\title{
PTF12os and iPTF13bvn
}

\section{Two stripped-envelope supernovae from low-mass progenitors in NGC 5806}

\author{
C. Fremling ${ }^{1}$, J. Sollerman ${ }^{1}$, F. Taddia ${ }^{1}$, M. Ergon ${ }^{1}$, M. Fraser ${ }^{2}$, E. Karamehmetoglu ${ }^{1}$, S. Valenti ${ }^{3}$, A. Jerkstrand ${ }^{4}$, \\ I. Arcavi ${ }^{3,5}$, F. Bufano ${ }^{6}$, N. Elias Rosa ${ }^{7}$, A. V. Filippenko ${ }^{8}$, D. Fox ${ }^{9}$, A. Gal-Yam ${ }^{5}$, D. A. Howell ${ }^{3,10}$, R. Kotak ${ }^{4}$, \\ P. Mazzali ${ }^{11,12}$, D. Milisavljevic ${ }^{13}$, P. E. Nugent ${ }^{8,14}$, A. Nyholm ${ }^{1}$, E. Pian ${ }^{15,16}$, and S. Smartt ${ }^{4}$
}

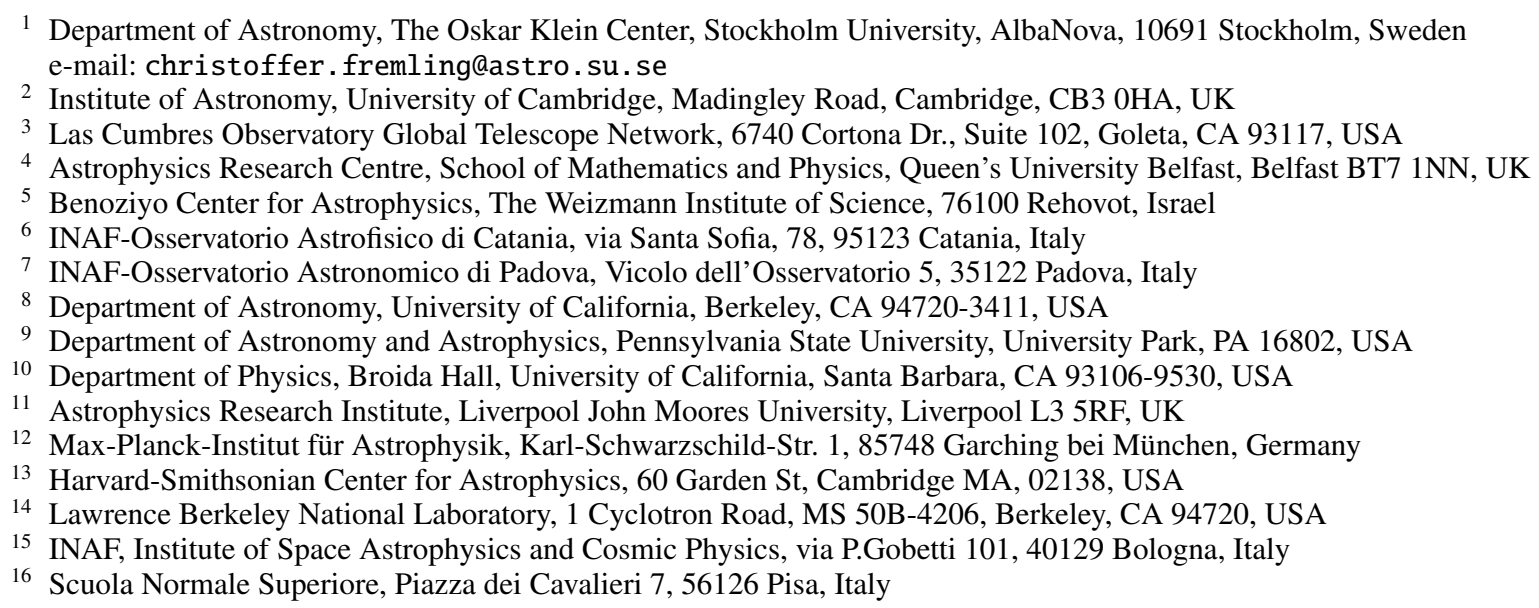

Received 8 February 2016/ Accepted 26 May 2016

\begin{abstract}
Context. We investigate two stripped-envelope supernovae (SNe) discovered in the nearby galaxy NGC 5806 by the (intermediate) Palomar Transient Factory [(i)PTF]. These SNe, designated PTF12os/SN 2012P and iPTF13bvn, exploded within 520 days of one another at a similar distance from the host-galaxy center. We classify PTF12os as a Type IIb SN based on our spectral sequence; iPTF13bvn has previously been classified as Type Ib having a likely progenitor with zero age main sequence (ZAMS) mass below $\sim 17 M_{\odot}$. Because of the shared and nearby host, we are presented with a unique opportunity to compare these two SNe.

Aims. Our main objective is to constrain the explosion parameters of iPTF12os and iPTF13bvn, and to put constraints on the SN progenitors. We also aim to spatially map the metallicity in the host galaxy, and to investigate the presence of hydrogen in early-time spectra of both SNe.

Methods. We present comprehensive datasets collected on PTF12os and iPTF13bvn, and introduce a new automatic referencesubtraction photometry pipeline (FPipe) currently in use by the iPTF. We perform a detailed study of the light curves (LCs) and spectral evolution of the SNe. The bolometric LCs are modeled using the hydrodynamical code HYDE. We analyze early spectra of both SNe to investigate the presence of hydrogen; for iPTF13bvn we also investigate the regions of the Paschen lines in infrared spectra. We perform spectral line analysis of helium and iron lines to map the ejecta structure of both SNe. We use nebular models and late-time spectroscopy to constrain the ZAMS mass of the progenitors. We also perform image registration of ground-based images of PTF12os to archival HST images of NGC 5806 to identify a potential progenitor candidate.

Results. We find that our nebular spectroscopy of iPTF13bvn remains consistent with a low-mass progenitor, likely having a ZAMS mass of $\sim 12 M_{\odot}$. Our late-time spectroscopy of PTF12os is consistent with a ZAMS mass of $\sim 15 M_{\odot}$. We successfully identify a source in pre-explosion HST images coincident with PTF12os. The colors and absolute magnitude of this object are consistent between pre-explosion and late-time HST images, implying it is a cluster of massive stars. Our hydrodynamical modeling suggests that the progenitor of PTF12os had a compact He core with a mass of $3.25_{-0.56}^{+0.77} M_{\odot}$ at the time of the explosion, which had a total kinetic energy of $0.54_{-0.25}^{+0.41} \times 10^{51} \mathrm{erg}$ and synthesized $0.063_{-0.011}^{+0.020} M_{\odot}$ of strongly mixed ${ }^{56} \mathrm{Ni}$. Spectral comparisons to the Type IIb SN 2011dh indicate that the progenitor of PTF12os was surrounded by a thin hydrogen envelope with a mass lower than $0.02 M_{\odot}$. We also find tentative evidence that the progenitor of iPTF13bvn could have been surrounded by a small amount of hydrogen prior to the explosion. This result is supported by possible weak signals of hydrogen in both optical and infrared spectra.
\end{abstract}

Key words. supernovae: general - supernovae: individual: PTF12os - galaxies: individual: NGC 5806 techniques: image processing - supernovae: individual: iPTF13bvn

* Tables 3-6 are only, and spectra are also, available at the CDS via anonymous ftp to cdsarc.u-strasbg. fr (130.79.128.5) or via http://cdsarc.u-strasbg.fr/viz-bin/qcat?J/A+A/593/A68 


\section{Introduction}

Prior to their final fate as core-collapse (CC) supernovae (SNe) releasing $\sim 10^{51}$ erg of kinetic energy, the progenitor stars of Type IIb and Ibc SNe have had their envelopes stripped of hydrogen. These SN types are therefore commonly referred to as stripped-envelope (SE) SNe.

In the case of SNe IIb, the stripping is partial, and earlytime spectra show clear signatures of Balmer lines. At later times the spectra of SNe IIb instead closely resemble those of Type Ib $\mathrm{SNe}$, which by definition do not show significant hydrogen signatures at any time (e.g., Filippenko 1997). Type Ic SNe likely experience even stronger stripping, resulting in a loss of their entire (or nearly entire) He envelope prior to explosion.

Two main mechanisms have been suggested to produce SE SNe: either binary mass transfer (e.g., Iben \& Tutukov 1985; Yoon et al. 2010; Claeys et al. 2011; Dessart et al. 2012; Yoon 2015) or strong line-driven winds from massive Wolf-Rayet (WR) stars (e.g., Conti 1976; Groh et al. 2013; Smith 2014).

Binary mass transfer is an appealing mechanism for producing the partial stripping seen in SNe IIb; when the envelope of the donor star decreases in radius below the Roche limit, the mass transfer naturally stops. Simulations of binary evolution show that the final amount of stripping of the exploding star depends mainly on the initial masses of the two stars in the binary system, the spatial configuration of the system, and the metallicity of the stars. Within the parameter space explored by Yoon et al. (2010) and others, both SNe IIb and Ib are produced in a bimodal fashion, but not at the observed rates (e.g., Li et al. 2011; see also Claeys et al. 2011).

It is also possible to produce both complete and partial stripping in a single-star scenario (e.g., Groh et al. 2013; Smith 2014). In this case the final amount of hydrogen depends strongly on the initial mass and metallicity of the isolated star, with higher metallicity allowing stronger line-driven winds and more mass loss. In this picture, SNe occurring in galaxies with low metallicity could end up as partially stripped $\mathrm{SNe}$ IIb, and $\mathrm{SNe}$ occurring in higher-metallicity environments could end up as completely stripped $\mathrm{SNe} \mathrm{Ib}$.

In the literature there is evidence (e.g., Kelly \& Kirshner 2012) for SNe IIb tending to reside in lower-metallicity hosts compared to $\mathrm{SNe} \mathrm{Ib}$. However, to produce completely stripped SN progenitors from single stars, progenitors with high zero age main sequence (ZAMS) masses of $30 M_{\odot}$ and beyond are typically needed. Such progenitors give rise to very large ejecta masses of $\sim 10 M_{\odot}$ as they explode. This is not consistent with the observed ejecta-mass range of 3.6-5.7 $M_{\odot}$ derived for most SNe Ibc by Taddia et al. (2015). In a binary scenario, a low ejecta mass is more easily produced since less-massive stars can end up completely stripped by the mass transfer. Similar low values for ejecta masses of SNe Ib/c were also deduced by Cano (2013) and Lyman et al. (2016).

Observational evidence for binarity in the progenitors of SNe IIb exists; for the well-known Type IIb SN 1993J, a binary companion was directly detected by Maund \& Smartt (2009); see also Fox et al. (2014). Further, the progenitor of the well-observed Type IIb SN 2011dh has been shown by Ergon et al. $(2014,2015)$ to most likely be a composite of a nearly completely stripped compact He core with a mass of 3.3-4.0 $M_{\odot}$ surrounded by a thin H-rich envelope extending out to $200-300 R_{\odot}$. Binary evolution modeling can readily reproduce these properties at the time of explosion, while single-star models encounter difficulties in reproducing the relatively low ejecta mass in combination with the observed $\mathrm{H}$ shell
(Claeys et al. 2011; Benvenuto et al. 2013). Recently, Folatelli et al. (2014a) have also claimed that the remaining flux in Hubble Space Telescope (HST) imaging obtained $\sim 1160 \mathrm{~d}$ past the explosion is consistent with a suitable binary companion to SN 2011dh; however, Maund et al. (2015) disagree with this conclusion.

The Type Ib SN iPTF13bvn was initially suggested to have been the result of the explosion of a single massive WR star based on model fits to the early photometry as well as on the colors and absolute magnitude of the progenitor candidate identified in pre-explosion HST images (e.g., Cao et al. 2013; Groh et al. 2013). However, hydrodynamical modeling and nebular $r$ band photometry indicate that the ZAMS mass was likely not more than $17 M_{\odot}$ (Fremling et al. 2014). This result was confirmed by the nebular-phase observations of Kuncarayakti et al. (2015). A single-star model with this ZAMS mass should not produce any kind of SE SN. This indicates that a binary was also the origin for this system. The observed colors in the preexplosion HST images of iPTF13bvn have since also been reassessed and reproduced by evolutionary modeling of a binary system (Bersten et al. 2014; Eldridge et al. 2015). Furthermore, the observables of iPTF13bvn are consistent with other SNe Ib in the literature (Taddia et al. 2015), which indicate that most SNe Ib have progenitors of similar nature. Independent photometry and spectroscopy of iPTF13bvn was also collected by Srivastav et al. (2014), showing consistent data and similar conclusions.

In this paper we present the first comprehensive dataset on PTF12os (SN 2012P), a SN IIb that occurred in the same nearby galaxy, NGC 5806, as the already well-studied Type Ib SN iPTF13bvn. We also supplement previously published data on iPTF13bvn with more photometry and spectra, and perform a comprehensive comparison of these two SNe. In addition, we compare their host environments via spectroscopic metallicity measurements of nearby $\mathrm{H} \alpha$ regions. Our analysis is aided by comparisons with SN 2011dh.

This paper is organized as follows. In Sect. 2 we discuss our observations and reduction procedures. A description of the reference-subtraction code that has been used to reduce most of our imaging is presented in detail. Section 3 gives long-slit metallicity measurements of $\mathrm{H} \alpha$ regions in NGC 5806 to determine the metallicity at the positions of iPTF13bvn and PTF12os and the metallicity gradient of the host galaxy. Extinction estimates for the two $\mathrm{SNe}$ are given in Sect. 4, and in Sect. 5 we discuss the result of astrometric identification of a progenitor candidate for PTF12os using HST pre-explosion images of NGC 5806. In Sect. 6 we present the multiband LCs of the $\mathrm{SNe}$ and construct pseudobolometric LCs of PTF12os and iPTF13bvn, which are then compared with SN 2011dh to get a first handle on the properties of the SN progenitors and explosions. In Sect. 7 we report our spectroscopy of the two SNe; we investigate the presence of early-time $\mathrm{H}$ in PTF12os, search for similar indications in the early spectra of iPTF13bvn, and perform spectral-line velocity measurements of the He and Fe lines to estimate the photospheric expansion velocities. The latter are used in Sect. 8 together with the hydrodynamical model HYDE (Ergon et al. 2015) to constrain the explosion parameters, such as the synthesized $\mathrm{Ni}$ and ejecta masses as well as the He-core mass of the progenitor of PTF12os. To allow direct comparison, we have recalculated the explosion parameters for iPTF13bvn and SN 2011dh using the same code. In Sect. 7 we also use late-time spectroscopy (>200 d past the explosions) of both iPTF13bvn and PTF12os to constrain the amount of oxygen in the ejecta of each SN by comparing our data to the nebular models of 
Jerkstrand et al. (2016). Finally, Sect. 9 contains a summary of this work, some discussion, and our conclusions.

\section{Observations and data reduction}

\subsection{Discovery and imaging}

The discoveries $^{1}$ of PTF12os (SN 2012P ${ }^{2}$ ) (2012 Jan. 10.48; Arcavi et al. 2012) and iPTF13bvn (2013 June 16.24; Arcavi et al. 2013a,b) in NGC 5806 were made with the Palomar Oschin 48-inch (P48) Schmidt telescope (Law et al. 2009). PTF12os was discovered $4.0 \mathrm{~d}$ past the explosion $\left(+4 \mathrm{~d}^{3}\right), t_{\exp }=2012 \mathrm{Jan}$. $6.50_{-1.3}^{+0.5}$, as estimated from the best fit to our hydrodynamical model grid (Sect. 8). The explosion date of iPTF13bvn is very well constrained ( $t_{\exp }=2013$ June 15.67; Cao et al. 2013) based on a power-law fit to the earliest $\mathrm{P} 48$ data points, which sets the discovery at $+0.6 \mathrm{~d}$.

Cao et al. (2013) reported early-time photometry on iPTF13bvn obtained with the P48, the robotic Palomar 60inch telescope (P60; Cenko et al. 2006), and the Las Cumbres Observatory Global Telescope network (LCOGT; Brown et al. 2013) up to $20 \mathrm{~d}$ past the discovery. Later follow-up data obtained with the same telescopes, along with the addition of nebular-phase photometry up to $\sim 240 \mathrm{~d}$ past discovery from the Nordic Optical Telescope (NOT), were reported by Fremling et al. (2014).

In this paper we provide final reductions in table format (Table 3) of the previously reported photometry from the P60, NOT, and LCOGT of iPTF13bvn (Cao et al. 2013; Fremling et al. 2014), along with previously unpublished late-time photometry obtained with the P60, NOT, and the Palomar 200-inch telescope (P200) through $\sim 350 \mathrm{~d}$ past discovery.

We also present P48, P60, NOT, Liverpool Telescope (LT), Gran Telescopio Canarias (GTC), and New Technology Telescope (NTT) multiband observations of PTF12os through $\sim 210 \mathrm{~d}$ past discovery (Table 4) along with ultraviolet (UV) photometry obtained with Swift (Roming et al. 2004) for both PTF12os (Table 5) and iPTF13bvn (Table 6).

\subsection{Imaging reductions and photometry}

The reductions of our multicolor LCs of PTF12os and iPTF13bvn from data obtained with the P48, P60, P200, NOT, LT, and GTC are based on our in-house reference imagesubtraction pipeline currently performing real-time automatic reductions of iPTF P60 data. This pipeline, while operating in automatic mode, uses mosaiced Sloan Digital Sky Survey (SDSS; Ahn et al. 2014) images for the subtraction of the host-galaxy contribution from each science frame taken with the P60. This allows us to quickly obtain host-subtracted magnitudes and colors of newly discovered transients, a valuable aid when deciding on follow-up strategy for a particular transient. The code can in principle operate on any imaging dataset as long as suitable data for producing a reference are available. In this paper it is used to obtain publication-quality host-subtracted magnitudes for both iPTF13bvn and PTF12os from all of our imaging data.

To reduce $\mathrm{P} 48$ data, we utilize deeply stacked $\mathrm{P} 48$ references generated from images obtained during the years before the explosion of PTF12os. For data from the P60, LT, GTC, NOT, and

1 All dates in this paper are given in Universal Time (UT).

2 SN 2012P was also independently discovered by amateur astronomers (Dimai et al. 2012).

3 Throughout this paper, we adopt the convention that a plus sign followed by a time implies a time past the estimated explosion date. the P200, deep stacks of P60 images are used as the references. For iPTF13bvn, the P60 dataset obtained on PTF12os is used to generate the reference images, and vice versa. Since the two SNe happened more than $500 \mathrm{~d}$ apart and they both peaked at $\sim 16$ mag in the $r$ band, any possible remaining flux from the $\mathrm{SNe}$ in the reference frames is negligible.

The top layer of the pipeline is written in MATLAB ${ }^{4}$, operating on images that are bias-subtracted and flat-fielded. The basic steps performed by this pipeline are described in sequential order below. When relevant, we also give some details pertaining to the quality of the reductions of our data on PTF12os and iPTF13bvn presented in this paper.

\subsubsection{The Fremling Automated Pipeline, FPIPE}

- Quality control of the science data and bad-pixel masking: before any further processing and image subtraction, the pipeline checks for the presence of a World Coordinate System (WCS) solution and its reliability. If no WCS is present, an astrometric solution is attempted. The approximate seeing and the number of stars present in each frame are also measured using SEXTRACTOR (Bertin \& Arnouts 1996). Any frames for which it is not possible to determine a robust WCS are discarded. If there is a known map of bad pixels for the CCD in use (e.g., the P60), these are also fixed by linear interpolation in this step.

- Sky subtraction: following the quality control, the sky background is removed from the images by masking out all detected sources (point-like or otherwise, using SEXTRACTOR with a low detection threshold), fitting a second-order polynomial surface with a first-order cross term to the remaining data in two iterations with $3 \sigma$ clipping, and then subtracting.

- Reference image generation: if a reference image is not manually specified, or if SDSS references are not used, a stacked reference is generated from suitable reference data as follows. Initially, images with seeing worse than $1.8^{\prime \prime}$ and images where there are a smaller number of detected stars than one third of the average in the dataset are filtered out. The remaining frames are sky subtracted, registered, matched in intensity, and combined using the average of all images with $3 \sigma$ clipping for each pixel. The sky subtractions are performed as described above and the registrations are performed as described below, using the image with the best seeing as the reference onto which the rest of the images are stacked.

- Image registration: to register the science images to the reference image, the centroids of common point sources in the reference and science frames are first identified using SEXTRACTOR. The geometric transformation is subsequently determined with different complexity depending on the number of common point sources. If there are fewer than 7 common point sources, the transformation allows for shifting, rotation, and scaling of the science image. If there are 7 to 15 common sources we also allow for shearing. With more than 15 common sources, which is typically the case, a second-order polynomial transformation with 12 parameters is determined, and the polynomial transformation is finally applied using Lanczos resampling. When operating on P60 references and science frames, the standard deviation in the distance between the centroids in the reference and a registered science frame is typically below 0.05 pixels. Frames

4 We also use the astronomy \& astrophysics package for MATLAB (Ofek 2014). 
with fewer than 3 point sources in common with the reference are discarded.

- Point-spread-function (PSF) modeling: to obtain a model for the PSF in the reference frame and in each science frame, we use SEXTRACTOR in combination with PSFEX (Bertin 2013). The current version of the pipeline uses a nonparametric PSF model, as measured from the raw data by cleaning and stacking the isolated point sources. We assume that the PSF is constant across the $\mathrm{CCD}^{5}$, an assumption that we have found to work very well for datasets solely obtained by the P60 or data from the P60 in combination with SDSS references, the main use of this pipeline. We find no evidence for a spatial dependence on the subtraction residuals in our subtracted frames.

- PSF matching: once the PSFs of the reference and each science frame have been determined, we convolve the reference image with the PSF of the science frame, as well as the science frame with the PSF of the reference, for each individual subtraction. This method, also known as the common PSF method (CPM), has been previously proposed by Gal-Yam et al. (2008).

We find that this method is well suited for subtractions where the reference image is not necessarily obtained with the same telescope as the science observations, since there are no parameters to be tuned except the size of the box region used to measure the PSF. However, both the reference and the science frames are somewhat degraded owing to convolution being performed on both images. Finally, the PSF models themselves are also convolved with each other to obtain the final PSF in each subtraction for later use when performing PSF photometry.

- PSF photometry: to determine the counts of a point source, we perform weighted least-squares fitting of the convolved nonparametric PSF model to the data. The fit is weighted by the photon noise in the images, so that areas with increased signal from the source receive higher weights in the fit. Before the fit is performed, the PSF model is centered on the point source using Discrete Fourier Transform crosscorrelation.

- Zero-point determinations and intensity-scale matching of the reference and science data: whenever there is SDSS coverage, or other local standard stars with known magnitudes are available for the field, we perform PSF photometry as described above on the locations of these stars in both the science and reference frames for each subtraction. Sources for which the quality of the fit to the PSF model is below a certain threshold are excluded, and the remaining sources are matched to their known magnitudes to determine the zero points (ZPs) of the frames ${ }^{6}$. We do not apply any color terms. The ZP information is used to scale the counts of the convolved reference frame to the convolved science frame, resulting in a common $\mathrm{ZP}$ as determined from the science frame and data that are ready to be subtracted.

For our Sloan-filter data of iPTF13bvn and PTF12os, we use SDSS magnitudes to determine the ZPs. Typically at least 20 useable SDSS stars are present in each science frame,

\footnotetext{
5 For P48 data this assumption is not true. To handle this, we cut out a small portion around the transient within which the PSF can be assumed to be constant for these reductions.

6 In practice, the result is that only sources that are detected in the science frame are used when determining the $\mathrm{ZP}$ in both the reference and the science frame.
}

resulting in very precise $\mathrm{ZP}$ determinations, with standard deviations of the mean for the ZP typically $<0.01 \mathrm{mag}^{7}$.

To set the ZPs in our $B$-band images we use $g$ - and $r$-band magnitudes of SDSS stars within the fields with the magnitude conversions described by Jester et al. (2005).

For our P48 $R$-band data, we use SDSS $r$-band magnitudes to determine the ZPs. The P48 uses a Mould $R$-band filter, which is not identical to the SDSS $r$ band. However, we find that this method gives LCs that are consistent within approximately \pm 0.05 mag compared to our P60 $r$-band LCs that have been reduced and calibrated in the same way. Thus, we can make the assumption that the ZPs we find in this way converts our Mould $R$-band data into Sloan $r$-band data, at least to a first approximation. Since this accuracy is sufficiently good for the science performed in this paper, we have not applied any color-corrections when determining the ZPs or corrections for the filter differences and spectral shapes (S-corrections) when determining the magnitudes of the transients in any of the imaging filters used, unless otherwise stated $^{8}$.

- PSF photometry of the transient, error determination, and detection limits: after subtracting the scaled reference image from the science frame, we finally perform a PSF model fit at the expected location of the transient. If the quality of the fit is above the detection threshold, the result is used to determine the magnitude of the transient based on the flux and the $\mathrm{ZP}$ of the science frame. To determine the uncertainty of the measured flux we insert artificial transients with the measured flux, scattered in a circular pattern around one PSF size away from the real transient, in the unsubtracted science frame. The subtraction is redone for each artificial transient and we measure the uncertainty as the standard deviation in the flux of 35 artificial sources. If the quality of the initial fit is below the detection threshold, a limiting magnitude is determined by inserting artificial sources of increasing magnitude until fewer than $66.7 \%$ of the inserted sources are detected. The initial threshold has been tuned so that the results from this procedure represent $3 \sigma$ detection limits, by comparison to results from aperture photometry.

- Final uncertainty in the measured magnitude: the final error in the determined magnitude of the transient is taken as the statistical uncertainty determined from the artificial sources added in quadrature to the standard deviation of the mean when doing the $\mathrm{ZP}$ determination from the detected point sources with known magnitudes in the science frame. Typically, for iPTF13bvn and PTF12os, the standard deviation of the mean of the ZP in our data is below $0.01 \mathrm{mag}$, as mentioned above; consequently, the final error is generally dominated by the statistical uncertainty when measuring the flux of the transients.

\footnotetext{
7 For the data from the LT that we present in this paper, the ZP is in some cases difficult to determine in the science frames because of their low signal-to-noise ratio $(\mathrm{S} / \mathrm{N})$, resulting in $\mathrm{ZP}$ uncertainties up to 0.05 mag for some epochs.

8 We caution that for other fields, color-corrections could be needed when determining the ZPs. For other SNe, S-corrections could also be more important than what we find for the $\mathrm{SNe}$ in this paper. Convolving the normalized transmission curves of the filters mounted on the P60 along with standard Sloan filter-profiles on the spectra of PTF12os and iPTF13bvn typically result in S-corrections smaller than 0.1 mag. We also find that our data obtained from other telescopes are consistent with the P60 LCs at this level or better, when we have overlap between the LCs.
} 


\subsubsection{LCOGT photometry}

For the images of iPTF13bvn obtained by LCOGT, we estimate the galaxy contribution by fitting and subtracting a low-order surface, and then performing PSF-fitting photometry (Valenti et al., in prep.). The Sloan filter data were calibrated against a minimum of 10 SDSS (Ahn et al. 2014) stars in the field. The Johnson-Cousins UBVRI filter data were calibrated against Landolt standard stars (Landolt 1992) observed during photometric nights. We find that this procedure gives consistent LCs in the bands where we also have reference-subtracted P60 data $(g, r, i)$. Thus, the hostgalaxy contribution at the location of iPTF13bvn appears to be negligible after the galaxy is subtracted in this way.

\subsubsection{Reduction of Swift-UVOT photometry}

Both PTF12os and iPTF13bvn were observed with the UV Optical Telescope onboard Swift (UVOT; Gehrels et al. 2004; Roming et al. 2005). PTF12os was observed in 6 epochs from 2012 Jan. 14 to Jan. $29(+8.7$ d to +23.5 d), and iPTF13bvn in 10 epochs from 2013 June 17 to 2013 July $23(+2.0 \mathrm{~d}$ to $+37.8 \mathrm{~d})$. Photometry was obtained as described by Brown et al. (2009), and an aperture with radius $5^{\prime \prime}$ was used.

To estimate the host-galaxy contribution at the location of the SNe we use the deepest Swift observation obtained when observing PTF12os as the reference frame for iPTF13bvn, and vice versa. The flux at the position of the transients was measured with the same aperture size in the reference frames as in the science frames and the flux measured in the reference was subtracted from each detection to obtain host-subtracted magnitudes. In this paper we only include data in the $U, U V W 1$, and $U V M 2$ filters, even though more bands were observed. We report our host-subtracted Swift-UVOT photometry as AB magnitudes in Table 5 and Table 6, and it is also shown in Fig. 5. For one epoch of observations of iPTF13bvn, there were no significant detections in these filters after the host contribution was subtracted.

\subsection{Spectroscopic observations and reductions}

We obtained 19 epochs of low-resolution optical spectra of PTF12os, starting before peak luminosity at $4 \mathrm{~d}$ past discovery up until $211 \mathrm{~d}$ past discovery, when the SN has entered the nebular phase. These are listed in Table A.1 along with the telescopes and instruments that were used. The full spectral sequence is shown in Fig. A.1.

Optical and near-infrared (NIR) spectra of iPTF13bvn were obtained starting within $24 \mathrm{~h}$ after discovery. Spectra until $16 \mathrm{~d}$ past discovery were published by Cao et al. (2013), whereas Fremling et al. (2014) presented 6 additional later-time optical spectra obtained between 18 and $86 \mathrm{~d}$ after discovery. In this paper we provide our complete dataset, consisting of 26 epochs of optical spectra and 4 NIR spectra, including the previously published data. The earliest spectrum of iPTF13bvn (Milisavljevic et al. 2013a), obtained with the SALT telescope, is also included in our analysis. We have obtained several new spectra in the nebular phase, one using the NOT and the Andalucia Faint Object Spectrograph (ALFOSC) at $250 \mathrm{~d}$ past discovery, one with the Deep Extragalactic Imaging Multi-Object Spectrograph (DEIMOS; Faber et al. 2003) on Keck 2 at 344 d past discovery, one with the ESO Very Large Telescope using the FORS2 spectrograph at 346 d past discovery and one with the Intermediate dispersion Spectrograph and Imaging System (ISIS) at the William Herschel Telescope (WHT) at $376 \mathrm{~d}$ past discovery. We also present an additional NIR spectrum that was not published by Cao et al. (2013), obtained with the Folded-port InfraRed Echellette spectrograph (FIRE; Simcoe et al. 2013) on Magellan-Baade at $78 \mathrm{~d}$ past discovery. Our spectral data on iPTF13bvn are listed in Table A.2. The optical spectra are shown in Fig. A.2 and the NIR spectra are in Fig. A.3.

All spectra were reduced using standard pipelines and procedures for each telescope and instrument. For our nebular spectrum of PTF12os obtained $211 \mathrm{~d}$ past discovery, we use spectra of the underlying H II region from several years after the explosion to subtract the background continuum. All spectral data and corresponding information is available via WISeREP ${ }^{9}$ (Yaron \& Gal-Yam 2012).

\section{Host-galaxy properties}

NGC 5806 is a nearby spiral galaxy having SAB(s)b morphology. The spectroscopic redshift of the galaxy from the SDSS is $z=0.00449$. We adopt the distance modulus $\mu=32.14 \pm$ 0.20 mag (Tully et al. 2013), corresponding to a distance of $26.8_{-2.4}^{+2.6} \mathrm{Mpc}^{10,11}$. We note that since PTF12os and iPTF13bvn occurred in the same galaxy, we do not have to worry about systematic uncertainties in the relative distances of the two $\mathrm{SNe}$. The above distance results in a $B$-band absolute magnitude for NGC 5806 of $M_{B}=-20.12 \mathrm{mag}^{12}$. Adopting the values from NED, the major and minor diameters of NGC 5806 are $185^{\prime \prime} 40$ and $94^{\prime \prime} .554$, respectively, and the position angle is $170^{\circ}$. The morphological T-type is 3.0 according to the Third Reference Catalogue of Bright Galaxies (RC3; de Vaucouleurs et al. 1991).

A stack of HST/WFC images taken in filters F658N, F435W, $F 555 W$, and $F 814 W$ during 2004 is shown in Fig. 1, with the locations of the known transients in the galaxy marked. In addition to iPTF13bvn and PTF12os which we investigate in detail here, the Type IIP SN 2004dg (Smartt et al. 2009) and the SN impostor SN Hunt 248 (Mauerhan et al. 2015a; Kankare et al. 2015) (discovered in 2014) also occurred in this galaxy. SN 2004dg is present in the image stack shown in Fig. 1.

\subsection{Metallicity estimates}

We have mapped the metallicity of NGC 5806 via a spectroscopic programme conducted at the $\mathrm{NOT}^{13}$ within which we performed spectroscopy of $\mathrm{H}$ II regions in the galaxy using ALFOSC in long-slit mode. This dataset was also supplemented by two WHT spectra (see Fig. 1).

Our metallicity measurements are based on strong-line diagnostics using the N2 method (Pettini \& Pagel 2004), which utilizes the ratio of the [N II] $\lambda 6584$ and $\mathrm{H} \alpha$ lines to estimate the abundance of oxygen in the line-emitting region. We estimate

\footnotetext{
9 http://www. weizmann.ac.il/astrophysics/wiserep/

${ }^{10}$ When the uncertainties are taken into account, this value is consistent with the kinematic distance of $23.9 \pm 1.7 \mathrm{Mpc}$.

11 An older and more uncertain distance modulus of $\mu=31.76 \pm$ 0.38 mag (Tully et al. 2009) has previously been used extensively in the literature for work on iPTF13bvn. However, we point out that the updated distance modulus estimate of $\mu=32.14 \pm 0.2$ mag by Tully et al. (2013) is very close to the median value and standard deviation $(\mu=32.09 \pm 0.2 \mathrm{mag}$ ) of all the distance modulus estimates reported on NED.

${ }^{12}$ Total $B$-band magnitude from NED, not corrected for extinction.

13 Proposal ID 48-408, PI C. Fremling, conducted in service mode.
} 


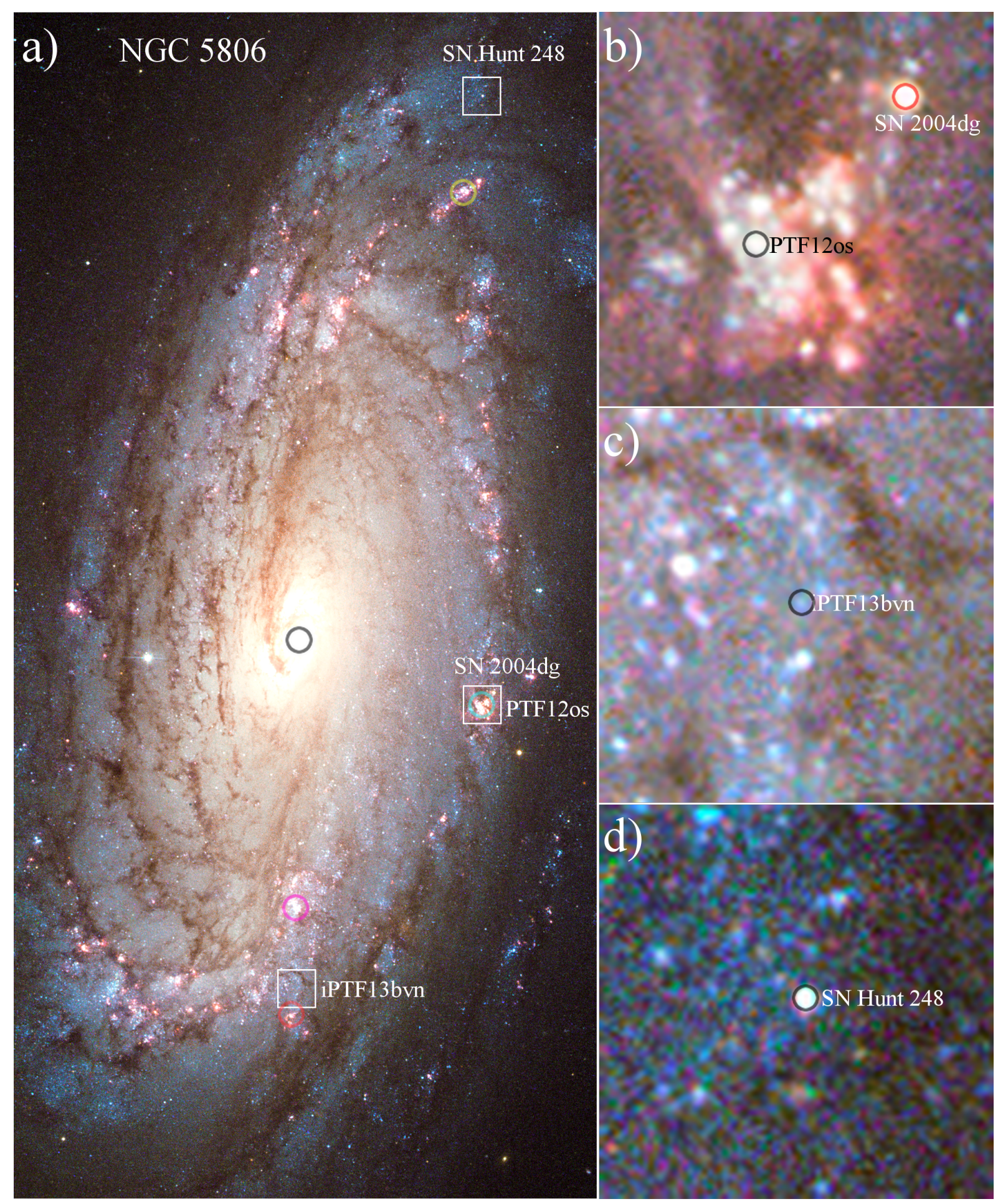

Fig. 1. NGC 5806 imaged by HST using WFC filters $F 435 W$, F555W, and $F 814 W$ during 2004 when SN 2004dg occurred in the galaxy. Regions with strong $\mathrm{H} \alpha$ emission $(F 658 N)$ are shown in red (Credits: ESA/NASA/Andre van der Hoeven). Panel a) shows the locations of PTF12os, PTF13bvn, SN 2004dg, and SN Hunt 248 marked by white boxes. Bright H II regions of which we have obtained spectra are indicated by colored circles. Panel b) displays a close-up view of the location of the PTF12os progenitor candidate (black circle). SN 2004dg, which exploded close to the position of PTF12os, is indicated by a red circle. Panel c) shows a close-up view of the location of iPTF13bvn, with the progenitor candidate marked by a black circle. Panel d) highlights the location of SN Hunt 248, which is marked by a black circle. North is up and east is to the left in all panels.

deprojected galactocentric radii $\left(r_{\mathrm{G}} / r_{25}\right)$ by following the procedures described by Taddia et al. (2013).

The location of PTF12os is coincident with a strong H II region, indicated by a blue circle in panel (a) of Fig. 1 (see also Sect. 5 for details on the progenitor identification). At this position we obtained the N2 metallicity estimate $12+\log _{10}(\mathrm{O} / \mathrm{H})=8.61 \pm 0.18 \mathrm{dex}$ using a NOT long-slit spectrum, and we measure the deprojected galactocentric radius 


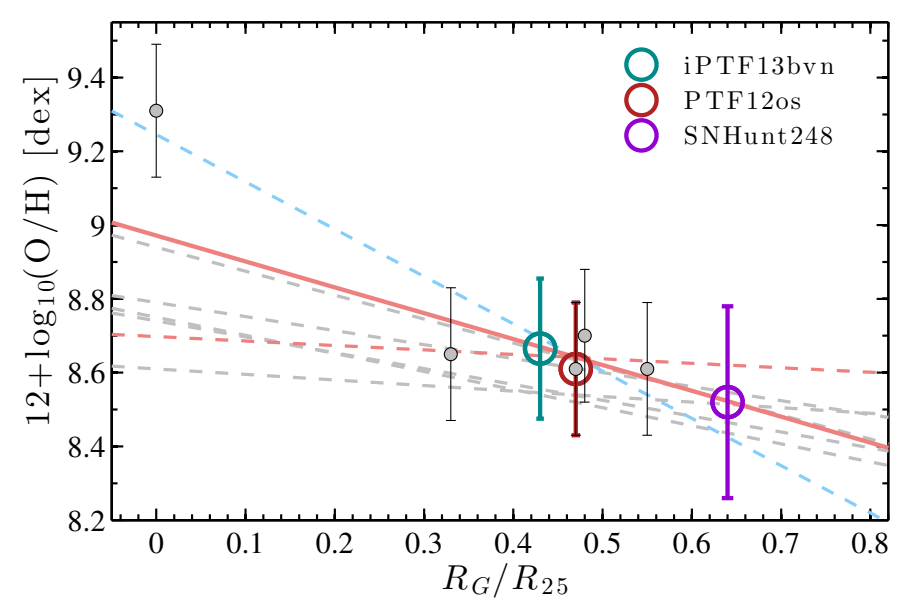

Fig. 2. Metallicity measurements (solid circles), metallicity estimates (open circles), and gradient estimate (solid red line) for NGC 5806 compared to the spiral-galaxy sample (gray dashed lines) by Gusev et al. (2012). The dashed blue line shows a first-order polynomial fit which includes the central oxygen metallicity measured for NGC 5806 using an SDSS spectrum, and the dashed red line shows the same fit with the metallicity datapoint at the center of the galaxy excluded.

$r_{\mathrm{G}} / r_{25}=0.47$. The same metallicity value was obtained at the position of the closest strong H II region to SN Hunt 248. This H II region is marked by a yellow circle in panel (a) of Fig. 1, and it is located at $r_{\mathrm{G}} / r_{25}=0.55$. SN Hunt 248 itself is situated to the north of this $\mathrm{H}$ II region at $r_{\mathrm{G}} / r_{25}=0.64$.

For the location of iPTF13bvn (see Fig. 1 and Sect. 5), we have estimated the deprojected galactocentric radius $r_{\mathrm{G}} / r_{25}=$ 0.43 , similar to that of PTF12os. With WHT/ISIS we obtained spectra of two bright $\mathrm{H}$ II regions close to this location, located at $r_{\mathrm{G}} / r_{25}=0.48$ (red circle in Fig. 1) and $r_{\mathrm{G}} / r_{25}=0.33$ (pink circle in Fig. 1). For these regions we estimated a N2 metallicity of $12+\log _{10}(\mathrm{O} / \mathrm{H})=8.70 \pm 0.18 \mathrm{dex}$ and $12+\log _{10}(\mathrm{O} / \mathrm{H})=$ $8.65 \pm 0.18$ dex, respectively.

To estimate the metallicity gradient in NGC 5806 we use the equation

$$
12+\log _{10}(\mathrm{O} / \mathrm{H})=12+\log _{10}(\mathrm{O} / \mathrm{H})_{0}+C_{\mathrm{O} / \mathrm{H}} \times\left(r_{\mathrm{G}} / r_{25}\right),
$$

where $12+\log _{10}(\mathrm{O} / \mathrm{H})_{0}$ is the central oxygen abundance and $C_{\mathrm{O} / \mathrm{H}}$ is the gradient (in dex $R_{25}^{-1}$ ). This equation fitted to the above data, including the central metallicity which we measure as $12+\log _{10}(\mathrm{O} / \mathrm{H})=9.31 \pm 0.18$ using an SDSS spectrum taken at the center of the galaxy, gives $12+\log _{10}(\mathrm{O} / \mathrm{H})=$ 9.25-1.28 $\left(r_{\mathrm{G}} / r_{25}\right)$ dex. This is a steeper gradient than the average for spirals (e.g., Pilyugin et al. 2004; Gusev et al. 2012). However, we note that in the above fit we are using the central oxygen abundance, without accounting for the possibility of AGN activity at the center of the galaxy. We see no clear evidence of an AGN in the spectrum, but a weak effect on the lines used in the abundance calculation cannot be excluded. If only $\mathrm{H}$ II regions in the disk are used, and the central abundance is extrapolated, we find the gradient in the disk of NGC 5806 to be $C_{\mathrm{O} / \mathrm{H}}=-0.12$ dex $R_{25}^{-1}$ and an extrapolated central oxygen abundance of $12+\log _{10}(\mathrm{O} / \mathrm{H})_{0}=8.70$ dex. The average of the two different gradients is

$$
12+\log _{10}(\mathrm{O} / \mathrm{H})_{\mathrm{NGC}} 5806=8.97-0.70\left(r_{\mathrm{G}} / r_{25}\right),
$$

and this is what we use as the best estimate for the metallicity gradient of NGC 5806. We show our measurements and gradients, along with the gradients of the sample of spiral galaxies studied by Gusev et al. (2012), in Fig. 2. The average gradient we find for NGC 5806 is in good agreement with this sample.

Given the metallicity gradient of NGC 5806 (Eq. (2)), the distance of iPTF13bvn from the center of the galaxy implies a metallicity of $12+\log _{10}(\mathrm{O} / \mathrm{H})=8.67 \pm 0.19 \mathrm{dex}$ at the position of the SN. At the position of SN Hunt 248 we find $12+\log _{10}(\mathrm{O} / \mathrm{H})=8.52 \pm 0.26 \mathrm{dex}$. To estimate the uncertainties we have accounted for the systematic error of 0.18 dex from the $\mathrm{N} 2$ method, as well as the difference between the two gradient fits and the average gradient at the position of each object.

In conclusion, it appears that both PTF12os and iPTF13bvn occurred in regions of very similar metallicity, where the oxygen abundance is close to the solar value $\left(12+\log _{10}(\mathrm{O} / \mathrm{H})=8.7 \mathrm{dex}\right.$; Asplund et al. 2009). The environment of SN 2011dh was also found to be roughly of solar metallicity by Van Dyk et al. (2011). However, a possible caveat here is that we do not have a direct measurement exactly at the position of iPTF13bvn, since the SN did not occur in an $\mathrm{H}$ II region which would allow the metallicity to be estimated using the N2 method.

\section{Extinction}

Throughout this paper all reddening corrections are applied using the Cardelli et al. (1989) extinction law with $R_{\mathrm{V}}=3.1$. For the Milky Way $(\mathrm{MW})$ color excess we adopt $E(B-V)_{\mathrm{MW}}=$ 0.0437 mag toward NGC $5806^{14}$ (Schlafly \& Finkbeiner 2011).

To estimate the host-galaxy color excess of PTF12os and iPTF13bvn we perform optical color comparisons to SN 2011 dh ${ }^{15}$, after correcting for the MW contributions. The multiband light curves (Sect. 6) were interpolated to evenly spaced dates in order to give one value for each night of observations, and then we used an iterative procedure that minimizes the following expression:

$\Delta_{\mathrm{C}}=\sum_{n=1}^{n=4} \sum_{t=10 \mathrm{~d}}^{t=30 \mathrm{~d}}\left[\left(C(n, E, t)_{\mathrm{SN}}-\left(C(n, t)_{\mathrm{dh}}\right)\right]^{2}\right.$,

where the sum over $n$ is performed for $C(n)=B-g, g-r, r-i$, and $i-z$ colors. $C(n, E, t)_{\mathrm{SN}}$ is also a function of $E(B-V)$ and time; it is the extinction-corrected color in a chosen set of filters as a function of time for the object for which the extinction is being computed. Similarly, $C(n, t)_{\mathrm{dh}}$ is the corresponding extinctioncorrected color for SN 2011dh.

If we assume that the extinction of SN 2011dh is known, we can now compute the host-galaxy color excess we need for our objects to get the best match to the colors of SN 2011dh. Here we adopt a total extinction $E(B-V)=0.07_{-0.04}^{+0.07}$ mag for SN 2011dh (Ergon et al. 2014). Effectively, this procedure minimizes the total difference in the $B-g, g-r, r-i$, and $i-z$ colors measured between $10 \mathrm{~d}$ and $30 \mathrm{~d}$ after explosion for a $\mathrm{SN}$ compared to SN 2011dh. These specific colors were chosen since we have the best-quality data in terms of cadence and uncertainties in these bands for both $\mathrm{SNe}$ simultaneously. For iPTF13bvn we find $E(B-V)_{\text {host }}=0.08_{-0.04}^{+0.07} \mathrm{mag}$, and for PTF12os we find $E(B-V)_{\text {host }}=0.29_{-0.05}^{+0.08} \mathrm{mag}$. The main assumption here is that these $\mathrm{SNe}$ have the same intrinsic colors (temperature) as SN 2011dh.

\footnotetext{
${ }^{14}$ We note that in Cao et al. (2013) the foreground (MW) extinction toward NGC 5806 was mixed up with the local extinction found at the position of iPTF13bvn. This mixup was also propagated to Fremling et al. (2014).

15 We use the LC data of SN 2011dh from Ergon et al. (2014, 2015).
} 
To estimate the total uncertainty intervals of these extinction values we have added in quadrature the statistical error resulting from the method described above to the error in the extinction of SN 2011dh. The statistical errors were derived via Monte-Carlo simulations by randomly applying $1 \sigma$ errors on all of the photometric data points used in the calculation and iterating the calculation a large number of times. The final errors are dominated by the uncertainty in the extinction estimate for SN 2011dh. Assuming another value for the extinction of SN 2011dh directly reflects in the calculated extinction values as a simple $1: 1$ relation.

We also checked the host-galaxy color excess of PTF12os by measuring the $\mathrm{NaI} \mathrm{D}$ equivalent width (EW) in the spectrum taken on 2012 Jan. 25. Using the relation suggested by Turatto et al. (2003),

$E_{(B-V)}=-0.01+0.16 \times E W(\mathrm{Na} \mathrm{ID})$,

we find $E(B-V)_{\text {host }}=0.265$ mag. This method is not very reliable since it is based on low-resolution spectra, and the Na I D feature is not resolved (see, e.g., Poznanski et al. 2011, 2012), but the presence of significant Na I D absorption confirms that the host extinction is not negligible. We note that two different slopes for the Na I D to $E_{(B-V)}$ relation were found by Turatto et al. (2003). We have adopted the shallower slope in Eq. (4). Using the relation derived by Poznanski et al. (2011) we find $E(B-V)_{\text {host }}=0.66 \mathrm{mag}$, which is consistent with what would be found by adopting the steeper slope found by Turatto et al. (2003). However, the scatter is very high in these relations (e.g. 0.3 mag in Poznanski et al. 2011), thus we consider the results from these relations as roughly consistent with each other as well as with our color-based method.

For iPTF13bvn, a host-galaxy color excess $E(B-V)_{\text {host }}=$ 0.0278 mag was previously derived from the Na I D absorption from high-resolution spectroscopy (Cao et al. 2013). A larger value of $E(B-V)_{\text {host }}=0.17 \pm 0.03$ mag was later suggested by Bersten et al. (2014) based on a preliminary $B-V$ color comparison to the Carnegie Supernova Project sample. Bersten et al. (2014) estimate $E(B-V)_{\text {host }}=0.07-0.22 \mathrm{mag}$ from another high-resolution spectrum. We note that the result from our color comparison to SN 2011dh is roughly consistent with all of these values when the uncertainties are taken into account.

\section{Progenitor identifications and photometry}

NGC 5806 has been comprehensively imaged with HST, both before and after the explosions of PTF12os and iPTF13bvn. We give a summary of the currently publicly available archival HST images covering the region of PTF12os in Table 1.

Moreover, images of iPTF13bvn were obtained in 2013 when this $\mathrm{SN}$ was present ${ }^{16}$, and they were used by multiple authors to confirm the progenitor candidate identification that was previously proposed from ground-based imaging (see Sect. 5). NGC 5806 was also reobserved at two epochs ${ }^{17}$ in 2015 in order to search for the binary companion of iPTF13bvn.

In this paper we use these observations to perform accurate astrometric registrations to locate a progenitor candidate of PTF12os and to constrain the mass of this candidate via its photometry (Sect. 5.1). For iPTF13bvn we do not perform any new analysis, but provide a summary of previous work (Sect. 5.2).

\footnotetext{
${ }^{16}$ GO-12888, PI S. Van Dyk, F555W. These images do not cover the explosion site of PTF12os.

172015 June 26 (see Table. 1) and 2015 June 30, GO-13822, PI G. Folatelli, F225W and $F 814 W$.
}

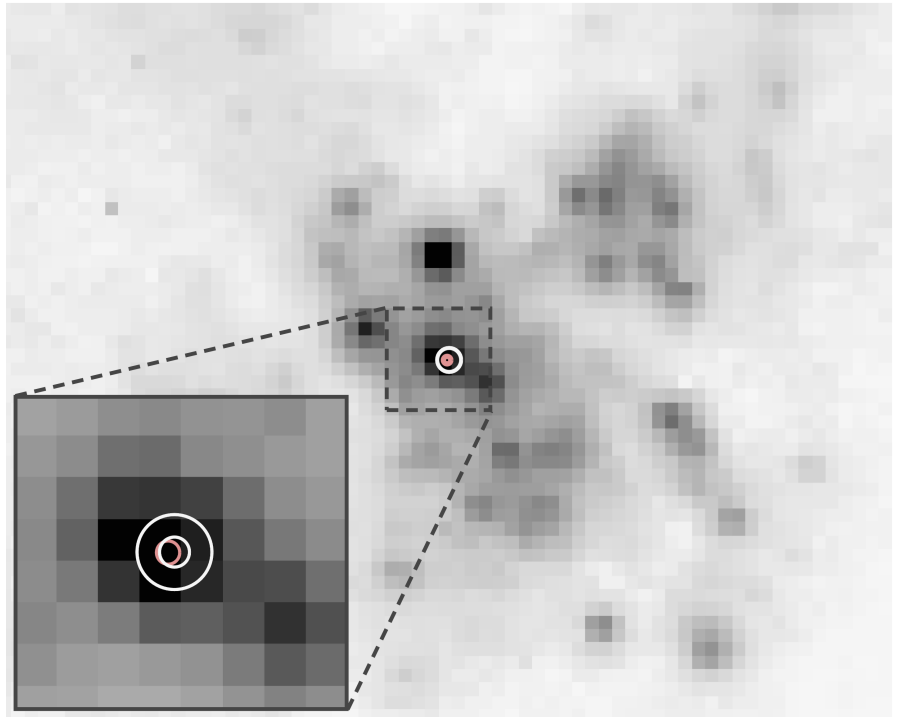

Fig. 3. Progenitor identification of PTF12os found by registering 8 ground-based NTT/EFOSC $i$-band images of PTF12os to a stacked preexplosion HST ACS F814W image. The field of view shown here is $44^{\prime \prime} 0 \times 22^{\prime \prime} 8$. North is up and east is to the left. The white circles show the average position of the centroid of PTF12os after image registration with a radius reflecting the statistical error from 7 image registrations. The smaller circle represents a $2 \sigma$ uncertainty and the larger circle represents a $5 \sigma$ uncertainty. The $2 \sigma$ uncertainty is only shown in the zoomed inset. The pink circles indicate the centroid of the possible progenitor measured in the HST image, with a radius representing the $2 \sigma$ uncertainty in its centroid.

\subsection{PTF12os}

A progenitor candidate for PTF12os was suggested by Van Dyk et al. (2012). To independently determine the location of the progenitor in NGC 5806, we obtained 11 -band images of PTF12os on 2012 Feb. $16(+42$ d) with the NTT at the La Silla Observatory in Chile. Using the 8 frames with the best seeing $\left(0 \prime .8-1{ }^{\prime \prime} 0\right)$, we perform astrometric registrations to a stacked (filters $F 814 W, F 555 W$, and $F 435 W$ ) archival HST (ACS/WFC) image from 2005 (Smartt et al. 2009) using 11 common point sources.

For the registrations we use a procedure based on standard MATLAB functions following the same principle as described by Fremling et al. (2014); the centroids of common point sources are derived from fits of two-dimensional (2D) Gaussians that allow for rotation, and the geometric transformation is determined as a second-order polynomial transformation with 6 free parameters and applied using bicubic interpolation.

After we performed the 8 registrations we applied $3 \sigma$ clipping to the values found for the position of the progenitor. This resulted in one calculation being excluded, leaving us with 7 values for the progenitor position. As the final position we then used the mean of these values and as the uncertainty we used the standard deviation in the mean, giving us a final uncertainty of 0.179 ACS/WFC pixels or 8.9 mas in right ascension $(\alpha)$ and 0.176 pixels or 8.8 mas in declination $(\delta)$.

We find that it is possible to constrain the progenitor location to the central source of a bright $\mathrm{H}$ II region (see Figs. 3 and 1) located at $\alpha=14^{\circ} 59^{\prime} 59^{\prime \prime} .082, \delta=+01^{\circ} 53^{\prime} 23^{\prime \prime}$ 67 (J2000.0) in the HST image. The centroid of this source, again determined by fitting a 2D Gaussian with rotation in the HST image, is offset by only 0.15 pixels or 7.7 mas from the progenitor location that we obtained from our registrations. Furthermore, the $2 \sigma$ uncertainty 


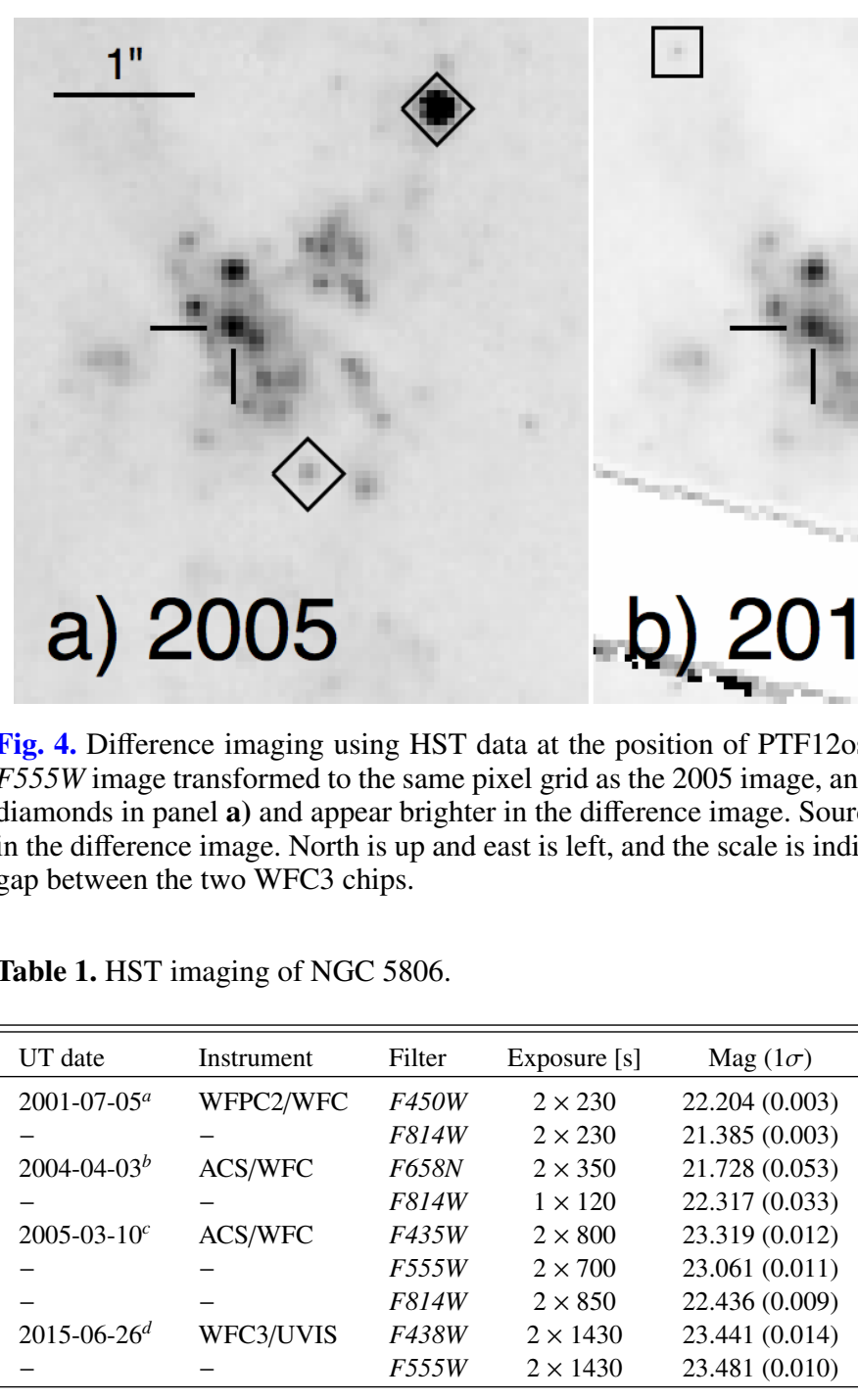

Notes. ${ }^{(a)}$ SNAP-9042, PI S. Smartt ; ${ }^{(b)}$ SNAP-9788, PI L. Ho ; ${ }^{(c)}$ GO10187, PI S. Smartt ; ${ }^{(d)}$ GO-13684, PI S. Van Dyk.

in the progenitor position that we find almost completely overlap with the $2 \sigma$ uncertainty in the centroid of the HST source (Fig. 3). Thus, we conclude that they are coincident. This is the same source that was first reported as a potential progenitor candidate by Van Dyk et al. (2012).

HST photometry of this progenitor candidate is shown in Table 1. The ACS/WFC photometry based on the images obtained in 2005 transformed to $B V I$ filters via a blackbody (BB) fit to the HST filters and by performing synthetic photometry using standard $B V I$ filter profiles are $B=23.30 \pm 0.01 \mathrm{mag}$, $V=22.94 \pm 0.01 \mathrm{mag}$, and $I=22.47 \pm 0.01 \mathrm{mag}$. After corrections for the distance modulus of NGC 5806 and the total extinction we get absolute magnitudes $M_{\mathrm{B}}=-10.18 \pm 0.20 \mathrm{mag}$, $M_{V}=-10.24 \pm 0.20 \mathrm{mag}$, and $M_{\mathrm{I}}=-10.29 \pm 0.20 \mathrm{mag}$, as well as colors $B-V=0.06_{-0.09}^{+0.06} \mathrm{mag}$ and $V-I=0.05_{-0.11}^{+0.07} \mathrm{mag}$. In the error bars for the colors we include the uncertainty in the extinction estimate.

PSF-fitting photometry was performed on the WFPC2 data using the HSTPHOT package (Dolphin 2000), and on the ACS and WFC3 images using DOLPHOT, a modified version of HSTPHOT. In all cases, images were masked using the data-quality files, the sky background measured, and library PSFs fitted to sources detected in each frame. The magnitudes were calibrated to the HST
Vegamag system using standard ZPs, and in the case of WFPC2 and WFC 3 the data were corrected for charge-transfer losses (the ACS images have already been corrected for this effect at the pixel level).

We find that the source coincident with PTF12os is consistently brighter in the WFPC 2 images. This is likely caused by the lower resolution of these data leading to blending with nearby sources. We therefore do not include the WFPC2 data in our further analysis. The two epochs of ACS imaging both include $F 814 W$ data, and we find a comparable magnitude for the progenitor in these two epochs. The magnitudes we measure are consistent with those reported by Van Dyk et al. (2012).

We also find consistent magnitudes between the ACS F435W image taken on 2005 March 10 and the WFC3 F438W image taken after the SN had faded on 2015 June 26. The fact that the source is still present at late times, coupled with the relatively bright absolute magnitudes, clearly suggest that this is a cluster rather than a single stellar progenitor of PTF12os.

The $F 555 \mathrm{~W}$ late-time magnitude measured in the postexplosion WFC3 image is $\sim 0.4$ mag fainter than what was measured in 2005. While it is appealing to interpret this as a deficit of flux owing to the disappearance of a progenitor, it is difficult to reconcile this with the lack of change in $F 435 W / F 438 W$. To further investigate this, we performed difference imaging using the 2005 and 2015 F555W images. The latter was registered to the former, before the two images were convolved to match their PSFs and scaled using the HOTPANTS package ${ }^{18}$. The result of this subtraction is shown in Fig. 4. No source can be seen at the position of PTF12os, suggesting no major change in flux between the two epochs. The flux at the position of PTF12os in this pre-explosion image cannot have been dominated by a single massive SN progenitor. In the precursor study by Strotjohann et al. (2015), it was also found that the source located at the position of PTF12os is still at approximately the same brightness 3 years after the SN occurred.

\footnotetext{
${ }^{18}$ http://wWw . astro. washington. edu/users/becker/v2.0/ hotpants.html
} 
Under the assumption that the source coincident with PTF12os is a cluster, we fitted the pre-explosion photometry using the CHORIzos package (Maíz-Apellániz 2004). Starburst99 models (Leitherer et al. 1999) were fitted to the measured HST ACS $F 435 W, F 555 W$, and $F 814 W$ magnitudes from 2005, along with the narrow-band $F 658 N$ magnitude from 2004 . The metallicity was set to be solar, while the extinction law was fixed to $R_{\mathrm{V}}=3.1$. The extinction was allowed to vary within the range estimated in Sect. 4. The best fit is achieved for a cluster age $\sim 5.5$ Myr.

This would imply that the progenitor of PTF12os was relatively massive. Using the STARS models, this corresponds to a ZAMS mass of $\gtrsim 25 M_{\odot}$ (Eldridge \& Stanway 2009), since single stars less massive than this should not yet have exploded. This value is significantly higher than the $<15 M_{\odot}$ estimate of the progenitor mass from our nebular spectroscopy found in Sect. 7.3. However, we are cautious about attributing too much weight to the cluster age derived here given the limited (4-band) photometric data. For comparison, the host cluster age and progenitor mass derived for SN 2004dj by Vinkó et al. (2009) relied on measurements in $\sim 20$ different bandpasses. It is also quite possible that the cluster contains multiple stellar populations of differing ages, with the SN progenitor being part of an older population and with the flux in the optical being dominated by a younger population.

\section{2. iPTF13bvn}

A probable progenitor candidate for iPTF13bvn, located at $\alpha=15^{\circ} 00^{\prime} 00^{\prime \prime}$. $147, \delta=+01^{\circ} 52^{\prime} 53^{\prime \prime}$.19 (J2000.0), was first identified in pre-explosion HST images by Cao et al. (2013). In Fremling et al. (2014) we used post-explosion HST (WFC3) images $^{19}$ of iPTF13bvn to confirm this result, also illustrated in Fig. 1 here. With these data it was possible to rule out other sources to a $5 \sigma$ level. This result was yet again confirmed by Eldridge et al. (2015), who reanalyzed the same pre- and postexplosion HST images.

In Cao et al. (2013) the apparent magnitudes for this source were first reported. However, a reanalysis by Eldridge et al. (2015) resulted in somewhat different values; $F 435 W=25.80 \pm$ $0.12 \mathrm{mag}, F 555 W=25.80 \pm 0.11 \mathrm{mag}$, and $F 814 W=25.88 \pm$ $0.24 \mathrm{mag}$.

After correcting for the reddening and distance modulus, we find the absolute magnitudes ${ }^{20} \cdot M_{F 435 W}=-6.86 \pm 0.23 \mathrm{mag}$, $M_{F 555 W}=-6.74 \pm 0.23 \mathrm{mag}$, and $M_{F 814 W}=-6.47 \pm 0.31 \mathrm{mag}$. By fitting a BB to these magnitudes and performing synthetic photometry using standard $B V I$ filter profiles, we find $M_{B}=$ $-6.83 \pm 0.23 \mathrm{mag}, M_{V}=-6.78 \pm 0.23 \mathrm{mag}$, and $M_{I}=-6.73 \pm$ $0.31 \mathrm{mag}$, as well as color estimates $B-V=-0.05_{-0.16}^{+0.17} \mathrm{mag}$ and $V-I=-0.05_{-0.26}^{+0.27} \mathrm{mag}$, where the error bars on the colors include the uncertainty in the extinction estimate.

These colors and absolute magnitudes could be consistent with a single WR star (Eldridge et al. 2013; Groh et al. 2013). However, previous studies (Fremling et al. 2014; Bersten et al. 2014; Eldridge et al. 2015; Kuncarayakti et al. 2015) conclude that a binary system is more likely for the progenitor of iPTF13bvn, based on the low ejecta and oxygen masses. In

\footnotetext{
19 Obtained on 2013 Sep. 2.37, GO-12888, PI S. Van Dyk.

${ }^{20}$ Due to our choice of $\mu=32.14 \pm 0.2$ mag, these absolute magnitudes are higher compared to previous studies. However, the upper limits considered by e.g. Eldridge et al. (2015) are consistent within our uncertainties. In Eldridge \& Maund (2016) the higher distance modulus is also fully taken into account.
}

the binary model for the iPTF13bvn system (20 $M_{\odot}$ primary and $19 M_{\odot}$ secondary initial masses in a very close binary) by Bersten et al. (2014), the source in the pre-explosion HST images was predicted to be dominated by light from the primary, which implies that the system should be significantly fainter after the SN has faded. Further, based on the binary model grid used by Eldridge et al. (2015), the HST photometry was shown to possibly be consistent with a wide range of initial separations and masses (10-20 $M_{\odot}$ for the SN progenitor). However, in the recent study by Eldridge \& Maund (2016), based on the HST observations obtained on 2015 June $26(+740 \mathrm{~d})$, it is shown that the object at the position of iPTF13bvn has dimmed, and is now fainter than in the pre-explosion images, confirming that the progenitor identification was correct. A similar conclusion was also made by Folatelli et al. (2016). While Eldridge \& Maund (2016) concluded that the observations were likely still dominated by light from the $\mathrm{SN}$ at the time of these observations, the mass range of the progenitor could be narrowed down significantly to $10-12 M_{\odot}$. A range that is consistent with our nebular models of iPTF13bvn, discussed in Sect. 7.3.

\section{Light curves}

The observed light curves (LCs) in Johnson/Cousins/Bessel $U B V R I$, SDSS griz and Swift $U V W 1$ and $U V M 2$ filters for PTF12os and iPTF13bvn along with SN 2011dh are shown in Fig. 5. All of the data in Fig. 5 have been corrected for distance and reddening according to Sects. 3 and 4 .

At first glance, the LCs of these three SNe appear surprisingly similar across all photometric bands, but a more detailed look does bring out some minor differences between them. PTF12os peaks in the $g$ band at approximately $+16 \mathrm{~d}$, iPTF13bvn at $+16.5 \mathrm{~d}$, and SN 2011 dh at $+20 \mathrm{~d}$ (see Fig. 6 for close-up view of the $g$ - and $r$-band LC peaks). The widths of the LC peaks are also slightly different. In the $r$ and $i$ bands especially, the LCs of iPTF13bvn appear to be somewhat narrower than those of both PTF12os and SN 2011dh. The width of the LC peak in $r$ is $~ 35$ days for iPTF13bvn and 50 days for PTF12os and SN 2011dh. This width was measured by finding the point on the rising part of the LC that is 1.5 mag fainter than the maximum, and then measuring the time until the same magnitude is reached on the declining part of the (interpolated) LC (again, see Fig. 6). Using the same measure a similar difference is observed in the $i$ band. However, it is somewhat difficult to isolate the peaks in the LCs of SN 2011dh and PTF12os in the $i$ and $z$ bands. It is also difficult to assess when the LC peaks end and the LCs start to decline in a more linear fashion. For iPTF13bvn, the LC peaks are somewhat more easily discernible in both the $i$ and $z$ bands.

Another minor difference can be seen in the apparent lack of a plateau phase immediately following the peak $(+50 \mathrm{~d})$ in bands bluer than the $r$ band for iPTF13bvn compared to PTF12os. PTF120s shows a temporarily slower decline between approximately $+50 \mathrm{~d}$ and $+100 \mathrm{~d}$ in $g$ and a virtually constant magnitude in $B$. For PTF12os we measure the $g$-band decline rate in this phase to be $0.011 \pm 0.002 \mathrm{mag} \mathrm{d}^{-1}$, based on a linear (firstorder polynomial) fit. For SN $2011 \mathrm{dh}$ we find a very similar decline rate of $0.013 \pm 0.001 \mathrm{mag} \mathrm{d}^{-1}$. For iPTF13bvn we measure $0.015 \pm 0.002 \mathrm{mag} \mathrm{d}^{-1}$. In the fit for iPTF13bvn, data points between $+50 \mathrm{~d}$ and $+80 \mathrm{~d}$ were used, since after $+80 \mathrm{~d}$ there is a gap in our photometric coverage until $+210 \mathrm{~d}$. However, our late-time data points between $+210 \mathrm{~d}$ and $+300 \mathrm{~d}$ indicate it is likely that the same decline rate as measured between $+50 \mathrm{~d}$ and $+80 \mathrm{~d}$ continued up until $300 \mathrm{~d}$ past the explosion in the $g$ band. 


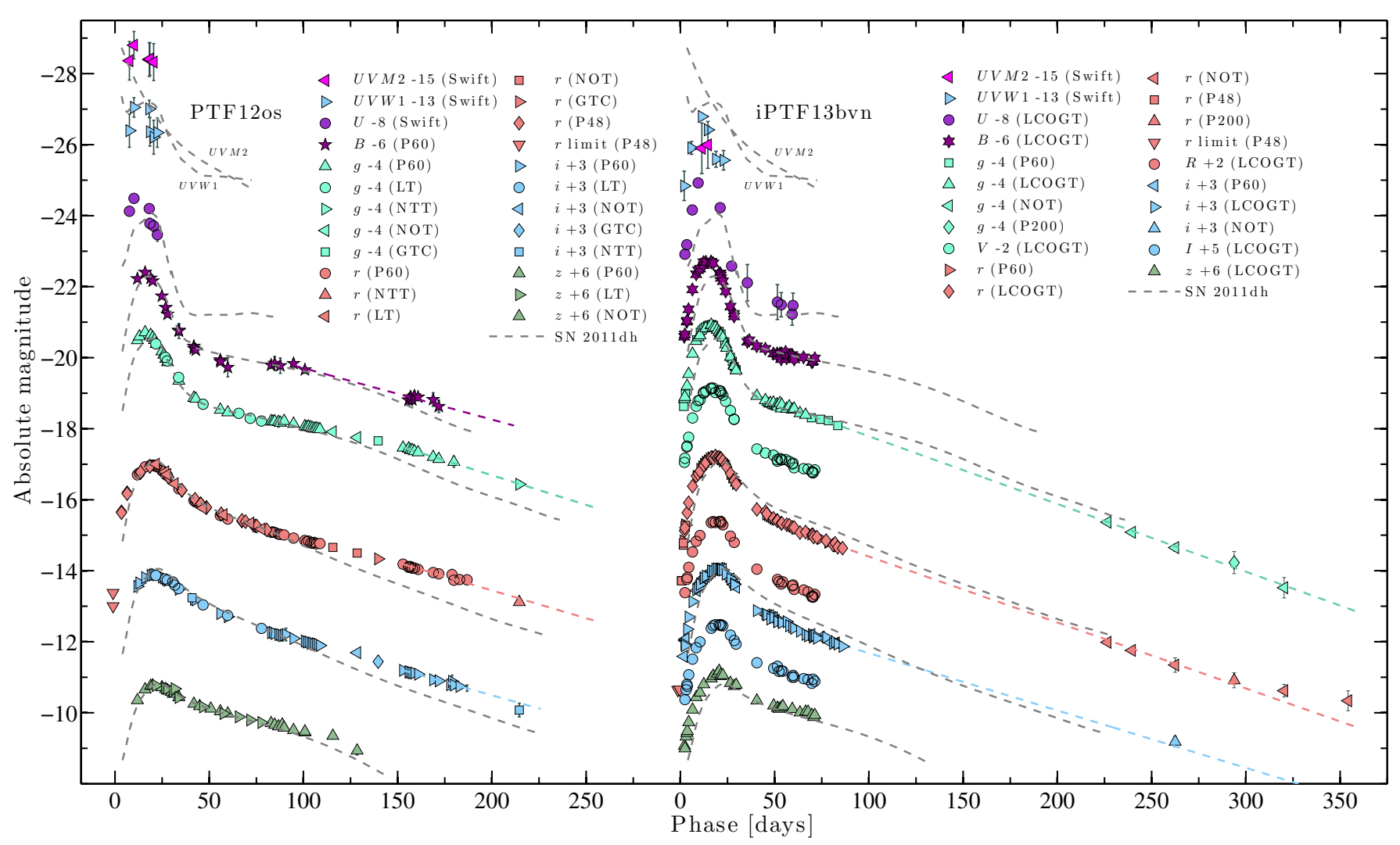

Fig. 5. Multicolor LCs of PTF12os (left) and iPTF13bvn (right) along with SN 2011dh (dashed black lines). The dashed colored lines represent first-order linear fits to the LC tails to estimate the late-time decline rates of the $\mathrm{SNe}$ (see Sect. 6). Corrections for extinction and distance have been applied according to Sects. 3 and 4. An S-correction based on a standard Johnson $U$-band filter has been applied to the $S w i f t U$-band data.

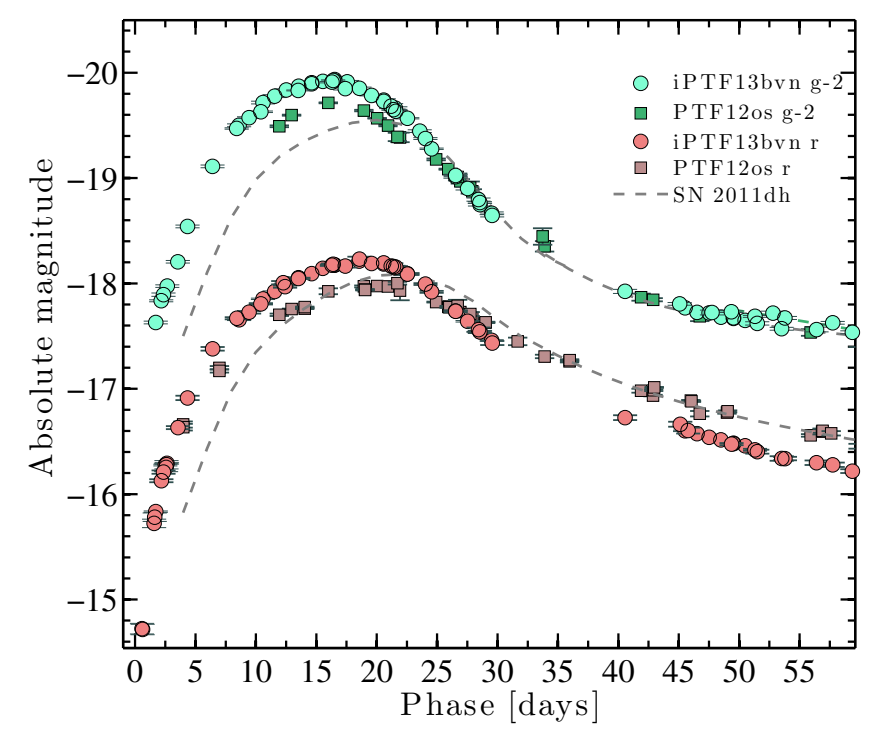

Fig. 6. Early-time $g$ - and $r$-band LCs of PTF12os (squares) and iPTF13bvn (circles) along with SN 2011dh (dashed black lines). Corrections for extinction and distance have been applied according to Sects. 3 and 4.

For the $B$ band we measure $0.000 \pm 0.002 \mathrm{mag} \mathrm{d}^{-1}$ for PTF12os, $0.010 \pm 0.001 \mathrm{mag} \mathrm{d}^{-1}$ for SN $2011 \mathrm{dh}$, and $0.010 \pm 0.003 \mathrm{mag} \mathrm{d}^{-1}$ for iPTF13bvn. For SN 2011dh this $B$-band decline rate continued until around $+125 \mathrm{~d}$, whereafter the decline rate increased significantly. This behavior is consistent with our data on PTF12os. However, for iPTF13bvn we lack coverage in the $B$ band past $+75 \mathrm{~d}$.
In the $U$ band, the data for both iPTF13bvn and PTF12os are not of sufficiently high quality to be able to discern whether there is a similar plateau as seen in the SN 2011dh data past $+50 \mathrm{~d}$. However, the photometric points for both PTF12os and iPTF13bvn are consistent with the photometry of SN 2011dh within the uncertainties. In the redder bands (riz), there is a similar trend as in the bluer bands in the decline rates between $+50 \mathrm{~d}$ and $+100 \mathrm{~d}$, with PTF12os showing marginally slower decline rates. In the riz bands we respectively measure $0.018 \pm 0.001 \mathrm{mag} \mathrm{d}^{-1}, 0.018 \pm 0.001 \mathrm{mag} \mathrm{d}^{-1}$, and $0.012 \pm$ $0.001 \mathrm{mag} \mathrm{d}^{-1}$ for PTF12os; $0.022 \pm 0.002,0.020 \pm 0.002$, and $0.016 \pm 0.003 \mathrm{mag} \mathrm{d}^{-1}$ for SN 2011dh; and $0.022 \pm 0.001$, $0.021 \pm 0.002$, and $0.011 \pm 0.003 \mathrm{mag} \mathrm{d}^{-1}$ for iPTF13bvn, based on similar first-order polynomial fits as described above.

While the widths of the LC peaks are somewhat different for iPTF13bvn and SN 2011dh, and iPTF13bvn lacks the plateau phase (at least in $g$ ) as discussed above, the decline rates become almost identical past $+150 \mathrm{~d}$ in all of the bands where our data allow this comparison to be made (gri; see the dashed lines in Fig. 5). For PTF12os, both the LC peaks and the late-time decline rates are very similar to those of SN $2011 \mathrm{dh}$, but owing to the slower declines immediately after the peak the fluxes in the $g, r$, and $i$ bands are somewhat higher past $+150 \mathrm{~d}$ compared to both SN 2011dh and iPTF13bvn.

To roughly estimate the post $+100 \mathrm{~d}$ decline rates of PTF12os and iPTF13bvn in the individual bands (the dashed and colored lines in Fig. 5), we performed first-order polynomial fits to the photometric data. For PTF12os we fit the data obtained between $+150 \mathrm{~d}$ and $+220 \mathrm{~d}$ in gri to avoid the plateaus in $g$ and $r$. Photometric data between $+70 \mathrm{~d}$ and $+300 \mathrm{~d}$ are used for iPTF13bvn. Based on these fits we find the late-time 


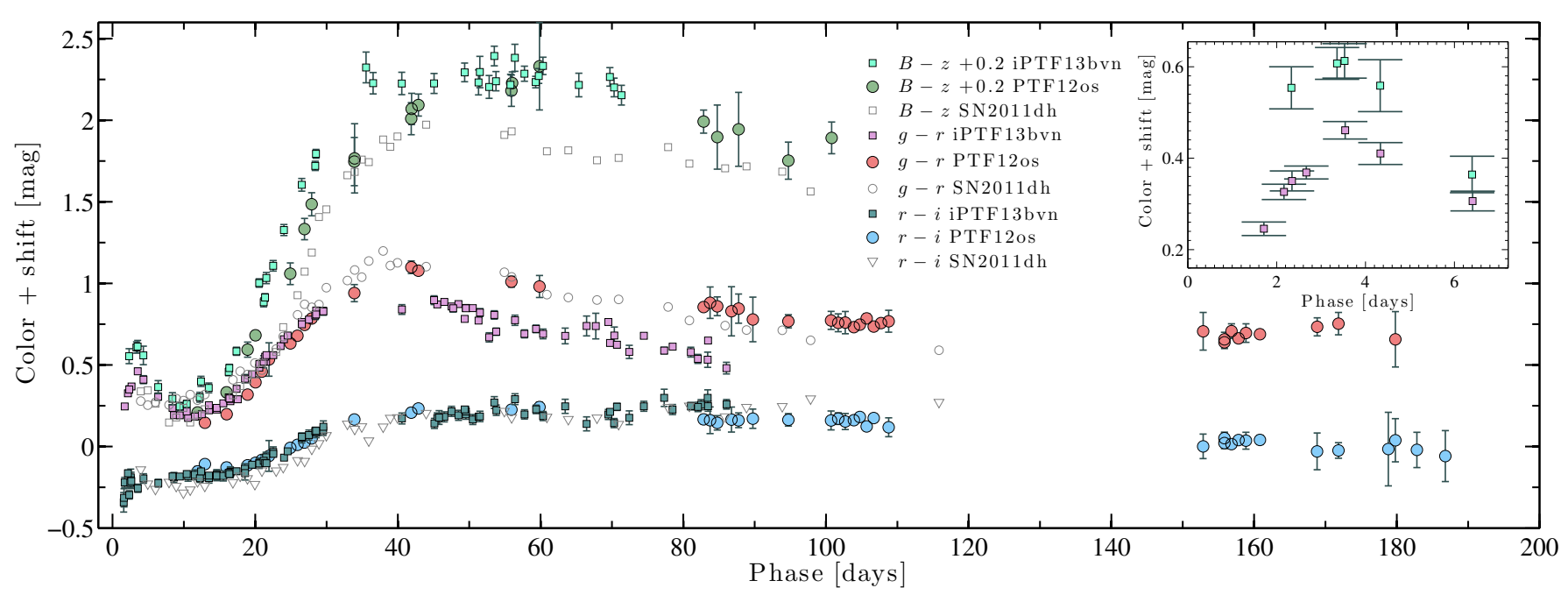

Fig. 7. Color evolution of PTF12os (solid colored circles) and iPTF13bvn (solid colored squares) along with SN 2011dh (open black markers). The photometry has been corrected for the extinction as described in Sect. 4. The error bars represent only the photometric uncertainty; we do not include the uncertainties in the extinction here.

decline rate in $r$ to be $0.019 \pm 0.001 \mathrm{mag} \mathrm{d}^{-1}$ for iPTF13bvn, and for PTF12os we measure a decline of $0.016 \pm 0.002 \mathrm{mag} \mathrm{d}^{-1}$. In $g$ we measure $0.019 \pm 0.001 \mathrm{mag}^{-1}$ for iPTF13bvn and $0.017 \pm 0.001 \mathrm{mag} \mathrm{d}^{-1}$ for PTF12os. In the $i$ band we measure $0.016 \pm 0.005 \mathrm{mag} \mathrm{d}^{-1}$ for iPTF13bvn and $0.017 \pm 0.001 \mathrm{mag} \mathrm{d}^{-1}$ for PTF12os. These decline rates are very similar to the decline rates of SN 2011dh in the corresponding bands at $+200 \mathrm{~d}$ (again, see Fig. 5). We note that we are by necessity fitting different time intervals for PTF12os and PTF13bvn, since there is a large gap in the coverage of iPTF13bvn past $+80 \mathrm{~d}$ and we lack photometry past $+220 \mathrm{~d}$ for PTF12os. This makes direct comparisons of the decline rates difficult. However, from Fig. 5, it is clear that PTF12os, overall, declined less from peak until at least +220 d compared to both SN 2011dh and iPTF13bvn, since the LC peaks are generally very similar in PTF12os while the latetime gri photometry is significantly brighter.

In the UV regime, the flux is comparable for PTF12os and SN $2011 \mathrm{dh}$ in both the $U V W 1$ and $U V M 2$ bands. For SN 2011dh a smooth decline was observed in the UVM2 band, while an initial decline followed by a peak was observed in the $U V W 1$ band $^{21}$. Our data on PTF12os are not of sufficiently high quality to determine whether this $\mathrm{SN}$ shows similar behavior. The first point in the $U V W 1$ band is not early enough, compared to the epochs where the initial decline was observed for SN 2011dh. Furthermore, the uncertainties of our UVM2-band data are too large to disentangle between a peak or a smooth decline in the observed LC. Compared to PTF12os and SN 2011dh, iPTF13bvn appears to have had a significantly lower UV flux. For this $\mathrm{SN}$ the first datapoint in the $U V W 1$ band was obtained very early, at around $+2 \mathrm{~d}$, but we still do not see the initial decline followed by a rise as for SN 2011dh. This could be interpreted as iPTF13bvn having a more compact progenitor compared to SN 2011dh, and hence a faster UV cooling tail following shock breakout (see also Sect. 6.2). In the $U V M 2$ band we only have two useable data points, and thus we cannot determine the LC shape. Finally, one should note that the UV regime is very sensitive to extinction corrections, and especially to the shape of the extinction law, which we simply assume here to be the same as for the MW (Sect. 4) for both objects. This, or

${ }^{21}$ We note that the peak observed in the $U V W 1$ band for SN $2011 \mathrm{dh}$ is likely a result of leakage from redder wavelengths. alternatively line blocking by metal lines, could also explain why the UV flux appears to be different in iPTF13bvn compared to PTF12os (and SN 2011dh). If the extinction law is too steep in the UV, the considerable extinction corrections we are using for PTF12os could result in an overestimated UV flux.

\subsection{Color evolution and blackbody fits}

A comparison of the color evolution in $B-z, g-r$, and $r-i$ for PTF12os, iPTF13bvn, and SN 2011dh is shown in Fig. 7. For these calculations the LCs have been interpolated to the dates of the bluest filter in each color. Since the multiband LCs are very similar, as previously discussed, all three SNe also appear similar in terms of the observed colors and color evolution. We note that this is in part by construction; we are matching the colors of PTF12os and iPTF13bvn to the colors of SN 2011dh between $+10 \mathrm{~d}$ and $+30 \mathrm{~d}$, to estimate the extinction (Sect. 4). However, other methods to calculate the extinction for both objects are consistent with the values that we have adopted, and the color evolution for each $\mathrm{SN}$ relative to the values between $+10 \mathrm{~d}$ and $+30 \mathrm{~d}$ is in any case not affected by our assumptions about the extinction.

The most apparent differences between these three SNe appear in the $B-z$ and $g-r$ colors. SN 2011dh temporarily shows a bluer color in $B-z$ by around 0.5 mag compared to PTF12os and iPTF13bvn, between $+50 \mathrm{~d}$ and $+70 \mathrm{~d}$. In $g-r$ the color is almost identical among the SNe up until $+30 \mathrm{~d}$, after which the color of iPTF13bvn plateaus at a $\sim 0.25$ mag bluer color of $g-r \approx 0.8 \mathrm{mag}$, indicating a significantly lower flux in the $g$ band, since the $r-i$ color continues to be similar also after $+30 \mathrm{~d}$. However, compared to the sample of SNe Ibc in Taddia et al. (2015), the $g-r$ color and time evolution in both PTF12os and iPTF13bvn appear consistent with those of other SNe Ib. The spread in the observed $g-r$ color in that sample after $+30 \mathrm{~d}$ is significantly larger than the difference observed between PTF12os and iPTF13bvn.

Another noteworthy difference is seen in the very early $g-r$ color of iPTF13bvn; up until approximately $+4 \mathrm{~d}$, a clear phase of gradual reddening in this color is observed (see the inset in Fig. 7). Similar behavior is seen to a lesser extent in the other colors. This could be evidence of a significant contribution from 


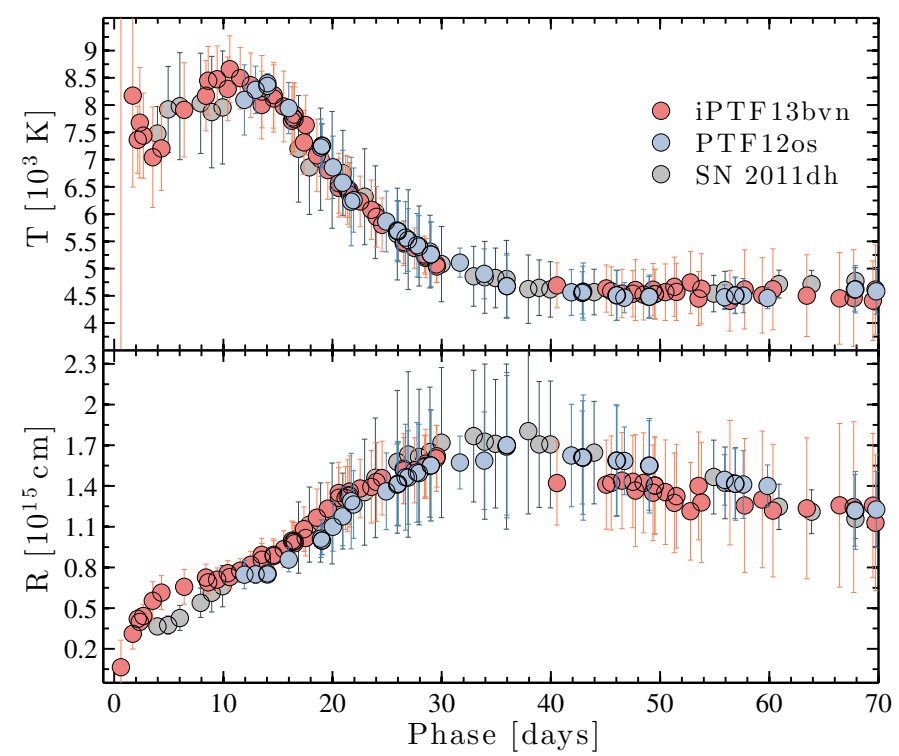

Fig. 8. Blackbody temperature (top panel) and radius (bottom panel) of iPTF13bvn (red circles), PTF12os (blue circles), and SN 2011dh (gray circles).

the cooling phase following shock breakout in the SN explosion, and it is discussed in more detail in Sect. 6.2.

We have determined the BB parameters of PTF12os, iPTF13bvn, and SN 2011dh by fitting BBs to the spectral energy distribution (SED) derived from the griz LCs (see Fig. 8). For these BB calculations we have interpolated the flux in the other bands to the dates of the $g$-band measurements. We correct for the distance and extinction according to Sects. 3 and 4.

Both in terms of the BB temperature and the radius, all three $\mathrm{SNe}$ appear to be very similar, except for the very early temperature evolution where iPTF13bvn shows signs of cooling in the earliest data. The BB temperature peaks at $\sim 8500 \mathrm{~K}$ for all three $\mathrm{SNe}$, and the radii peak at $\sim 1.7 \times 10^{15} \mathrm{~cm}$.

We note that the peak radius and uncertainties generally become smaller for SN 2011dh if the $r$ band is excluded from the BB fits. This indicates that the SED significantly deviates from a BB for SN 2011dh in $r$, which could possibly be explained by the relatively strong signature from hydrogen in the early spectra (Sect. 7). We also note that the radius found for SN 2011dh by Ergon et al. (2014) via fitting the VIJHK bands peaks at $\sim 1.4 \times 10^{15} \mathrm{~cm}$. That estimate should be more robust, since it covers a much larger wavelength range, and deviations from a $\mathrm{BB}$ in small parts of this range should not have as large an effect on the final result. A similar decrease in the BB radii of PTF12os and iPTF13bvn would be expected, given the similarity of all other LC bands. However, we do not have the needed wavelength coverage to perform that comparison.

\subsection{Early-time photometry}

For iPTF13bvn we have very early-time $r$-band photometry (at +0.6 d) from the P48, along with color information starting already from $+1.7 \mathrm{~d}$. In the early color evolution we observe a cooling trend up to around $+4 \mathrm{~d}$ in $g-r$ (see the inset of Fig. 7). Our BB fits to the griz filters (Fig. 8) show temperatures declining from $8500 \mathrm{~K}$ at around $+2 \mathrm{~d}$ to $7000 \mathrm{~K}$ at $+4 \mathrm{~d}$. Similar evolution is found when fitting BB SEDs to the earliest two spectra (see Sect. 7). In Fig. 9 we show the early BB temperature evolution, an estimated early bolometric LC, and the early gri photometry in detail.
One possible interpretation of these data is that we are at early times observing significant flux from the cooling phase following shock breakout (Piro \& Nakar 2013). Based on this assumption we have performed fitting of models with different radii to the early gri-band luminosity, the temperature evolution, and the early bolometric luminosity estimated from the BB temperature and radius using the Stefan-Boltzmann law. Our models are calculated according to Piro \& Nakar (2013, their Eqs. (1) and (2), henceforth abbreviated the PN1 model), and we also let the explosion date of iPTF13bvn be a free parameter in the fitting procedure.

We find that it is possible to fit the early temperature evolution with a model that is also consistent with the early photometry in the individual bands (the right panel of Fig. 9), by using a progenitor radius of $10 R_{\odot}$ and an explosion date of $t_{\text {exp }}=2013$ June 15.57. We note that after around $+2 \mathrm{~d}$, the luminosities in the individual bands start to greatly exceed what can be produced from a model based purely on shock-breakout cooling while still fitting the observed temperature (and estimated bolometric luminosity) simultaneously. We interpret this as the heating from radioactive nickel gradually becoming the dominant energy source.

For these calculations we have adopted the ejecta mass and total kinetic energy from our hydrodynamical model described in Sect. 8 and an opacity $\kappa=0.2 \mathrm{~cm}^{2} \mathrm{~g}^{-1}$. The radius we find here is significantly larger than what was found by Cao et al. (2013). This difference can be largely explained by a different approach; in Cao et al. (2013) it was assumed that the luminosity from the cooling phase should reach the plateau phase at a luminosity limited by the first photometric point observed by the P48 at $+0.6 \mathrm{~d}$. Here we assume that the cooling is still significant at least up until around $+2 \mathrm{~d}$. We are also using explosion parameters (ejecta mass, total kinetic energy) from hydrodynamical modeling, while Cao et al. (2013) adopted typical values for an SE SN. We note that the explosion date we find using this method is within $0.1 \mathrm{~d}$ of the explosion date estimate by Cao et al. (2013), but around $0.3 \mathrm{~d}$ later than the explosion date derived from our hydrodynamical modeling. We also note that a radius on the order of $10 R_{\odot}$ immediately prior to the explosion of an SE SN is expected in a binary scenario (see, e.g., Yoon et al. 2010).

From hydrodynamical simulations, Bersten et al. (2014) have argued that the early-time observations of iPTF13bvn could be consistent with a significantly larger radius, possibly even as large as $150 R_{\odot}$, if the explosion is assumed to have happened somewhat later than our estimate. That calculation was based only on the P48 $r$-band data. Using only the $r$ band and later explosion dates, we can also fit significantly larger radii. However, these models then become inconsistent with all of the remaining observations; too high temperatures compared to the observations are predicted, resulting in bad fits to the bolometric LC. However, there is evidence that analytical shock-cooling models tend to predict unrealistically high temperatures when the radius becomes large (e.g., Bersten et al. 2012). Lower temperatures in our models with larger radii and later explosion times could make them more consistent with the observed LCs. Thus, we do not consider the above radius estimate of $10 R_{\odot}$ as particularly robust, but it does seem unlikely that the progenitor would have had a significantly smaller radius. In the binary modeling by Eldridge \& Maund (2016) a radius on the order of $50 R_{\odot}$ is found for the progenitor of iPTF13bvn.

Finally, for PTF12os it is not possible to put any meaningful constraints on the progenitor radius based on a similar analysis, since the early P48 coverage does not start until around $+5 \mathrm{~d}$ and 

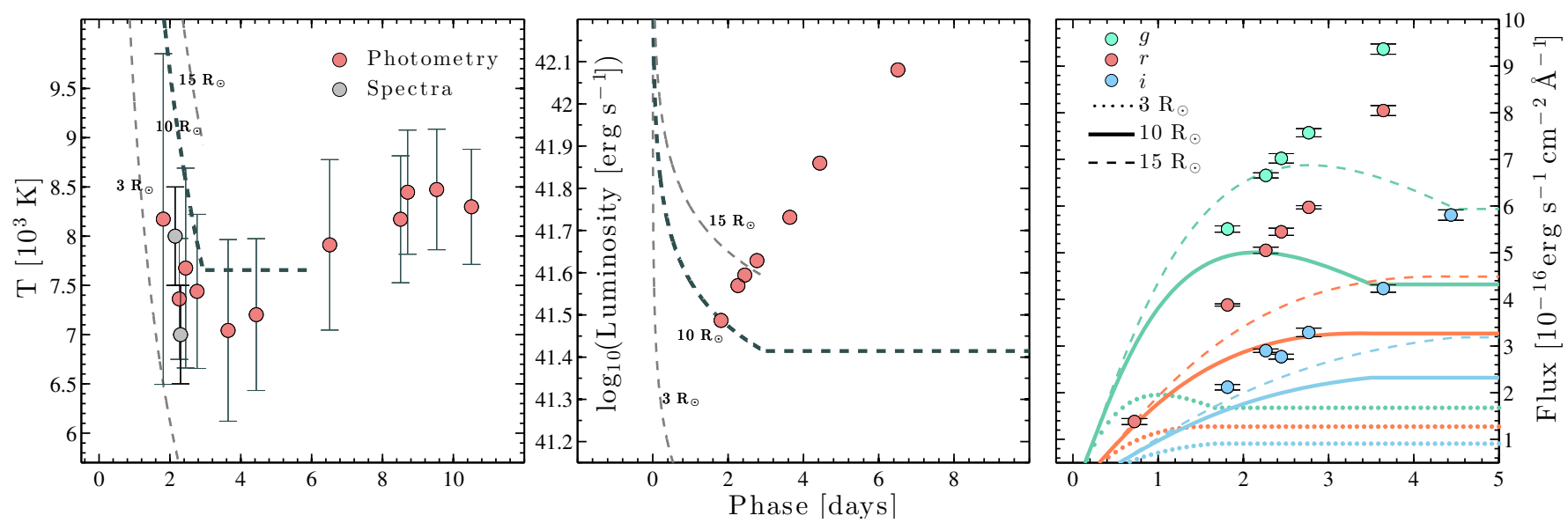

Fig. 9. Early BB temperature evolution of iPTF13bvn (left panel). Early bolometric luminosity calculated using the temperature evolution from the left panel and the Stefan-Boltzmann law (center panel). Early $g, r$, and $i$-band flux of iPTF13bvn (right panel). In each panel we also show the relevant quantity predicted by the PN1 models for the cooling emission after shock breakout for progenitor radii of 3,10 , and $15 R_{\odot}$. A model with a radius of $10 R_{\odot}$ is consistent with the observed data. In the left and center panels the $3 R_{\odot}$ and $15 R_{\odot}$ models are shown as thin dashed gray lines and the optimal model with a radius of $10 R_{\odot}$ is a thicker dashed black line. In the right panel the $3 R_{\odot}$ model LCs are displayed as dotted lines and the $15 R_{\odot}$ model LCs are thin dashed lines in matching colors to the photometry. The $10 R_{\odot}$ model LCs are shown as thicker solid lines.

we only have color information after $+10 \mathrm{~d}$. We stress the importance of obtaining early-time color information (ideally before $+2 \mathrm{~d}$ ) for constraining the radius of more SE SNe, following the above recipe or using hydrodynamical calculations.

\subsection{Quasi-bolometric light curves}

We have constructed quasi-bolometric LCs by integrating the flux in the Bgriz bands. For these calculations we have corrected for the distance and extinction according to Sects. 3 and 4. To determine the integrated fluxes we have interpolated the magnitudes in the other bands to the dates of the $r$-band measurements. We then converted the magnitudes into fluxes for each observed $r$-band epoch at the effective wavelengths of the filters and performed linear interpolation of these data at an even $1 \AA$ spacing, followed by integration between the effective wavelengths of the $B$ and $z$ bands.

We show a comparison of the Bgriz quasi-bolometric LCs of PTF12os, iPTF13bvn, and SN 2011dh in Fig. 10. Qualitatively, these LCs are again very similar for these three SNe, with only minor differences, as expected from the very similar multiband photometry. Similar to what was seen for the $g$ band, PTF12os and iPTF13bvn rise to peak in 16-18 d, while SN 2011dh rises to peak in $\sim 20 \mathrm{~d}$. The widths of the LC peaks also differ slightly, with the LC peak of iPTF13bvn being somewhat narrower, as was also seen in some of the individual LC bands. The bolometric LC peak width correlates with the ejecta mass of the explosion (Falk \& Arnett 1977); thus, this indicates that the ejecta mass could be slightly lower in iPTF13bvn compared with PTF12os and SN 2011dh, which should have very similar ejecta masses if only the LC peak widths are considered (but see Sect. 8 for a more comprehensive analysis based on our hydrodynamical models, which also account for the observed expansion velocities). The ejecta mass of SN $2011 \mathrm{dh}$ was estimated to be $\sim 2 M_{\odot}$, from previous hydrodynamical modeling (Ergon et al. 2014).

The quasi-bolometric peak luminosities are remarkably similar, with iPTF13bvn peaking at the highest luminosity of $10^{42.05} \mathrm{erg} \mathrm{s}^{-1}$, SN 2011dh peaking at $10^{42} \mathrm{erg} \mathrm{s}^{-1}$, and PTF12os at $10^{41.95} \mathrm{erg} \mathrm{s}^{-1}$. The peak luminosity is strongly correlated with the amount of radioactive nickel ejected in the explosions. Thus we find that these three SNe should have very similar nickel masses. In Fremling et al. (2014), the nickel mass of iPTF13bvn was found to be $\sim 0.05 M_{\odot}$, which is on the lower end of what is observed for typical SNe Ib/IIb. However, we have here adopted a distance modulus that is higher by $0.38 \mathrm{mag}$. The nickel mass of SN 2011dh $\left(\sim 0.075 M_{\odot}\right.$, Ergon et al. 2014) is typical for a SN IIb.

In the linear decline phase of the quasi-bolometric LCs, starting at approximately $+50 \mathrm{~d}$ for all three $\mathrm{SNe}$, the decline rates are very similar, when measured as linear (first-order polynomial) fits between $+50 \mathrm{~d}$ and $+70 \mathrm{~d}$. We find $0.019 \pm 0.001 \mathrm{mag} \mathrm{d}^{-1}$, for iPTF13bvn, $0.019 \pm 0.002 \mathrm{mag} \mathrm{d}^{-1}$ for PTF12os, and $0.020 \pm$ $0.003 \mathrm{mag} \mathrm{d}^{-1}$ for SN $2011 \mathrm{dh}$. After around $+80 \mathrm{~d}$, we observe a break in the LC of PTF12os toward a slower decline of $0.012 \pm 0.001 \mathrm{mag} \mathrm{d}^{-1}$, as measured by a linear fit between $+85 \mathrm{~d}$ and $+110 \mathrm{~d}$. This could indicate a more efficient trapping of $\gamma$-rays in the SN ejecta of PTF12os, as this decline rate is close to the expected decay rate of $\sim 0.01 \mathrm{mag} \mathrm{d}^{-1}$ from the energy deposition of radioactive ${ }^{56} \mathrm{Co}$ assuming complete trapping. This behavior is not seen in the LC of SN 2011dh, for which the same decline as measured between $+50 \mathrm{~d}$ and $+70 \mathrm{~d}$ continues for a significantly longer time, at least until around +300 d. However, Wheeler et al. (2015) show that the late-time decline rates ( $\gamma$-rays trapping efficiencies) among SE SNe can vary significantly. For iPTF13bvn, our photometric coverage in the $B$ and $z$ bands stops after $+70 \mathrm{~d}$, and thus we are not able to comment on the late-time Bgriz quasi-bolometric LC behavior. However, in Sect. 6 we found that the gri-band decline rates are very similar for iPTF13bvn and SN 2011dh also at much later times.

When constructing a quasi-bolometric LC of iPTF13bvn using methods similar to ours, Srivastav et al. (2014) found a rather steep post-peak decline rate of $\sim 0.03 \mathrm{mag} \mathrm{d}^{-1}$ between $+40 \mathrm{~d}$ and $+70 \mathrm{~d}$. This is a steeper decline compared with most other SNe IIb/Ib (Wheeler et al. 2015). However, the decline rate found by Srivastav et al. (2014) depended heavily on a single bolometric epoch at $+70 \mathrm{~d}$. Our quasi-bolometric LC of iPTF13bvn shows a slower decline rate, similar to that of SN 2011dh between $+50 \mathrm{~d}$ and $+70 \mathrm{~d}$. Our result is based on multiple epochs of Bgriz data during this phase, of which several are at around $+70 \mathrm{~d}$. Finally, we want to point out that although the quality of our photometry has allowed us to scrutinize minor 


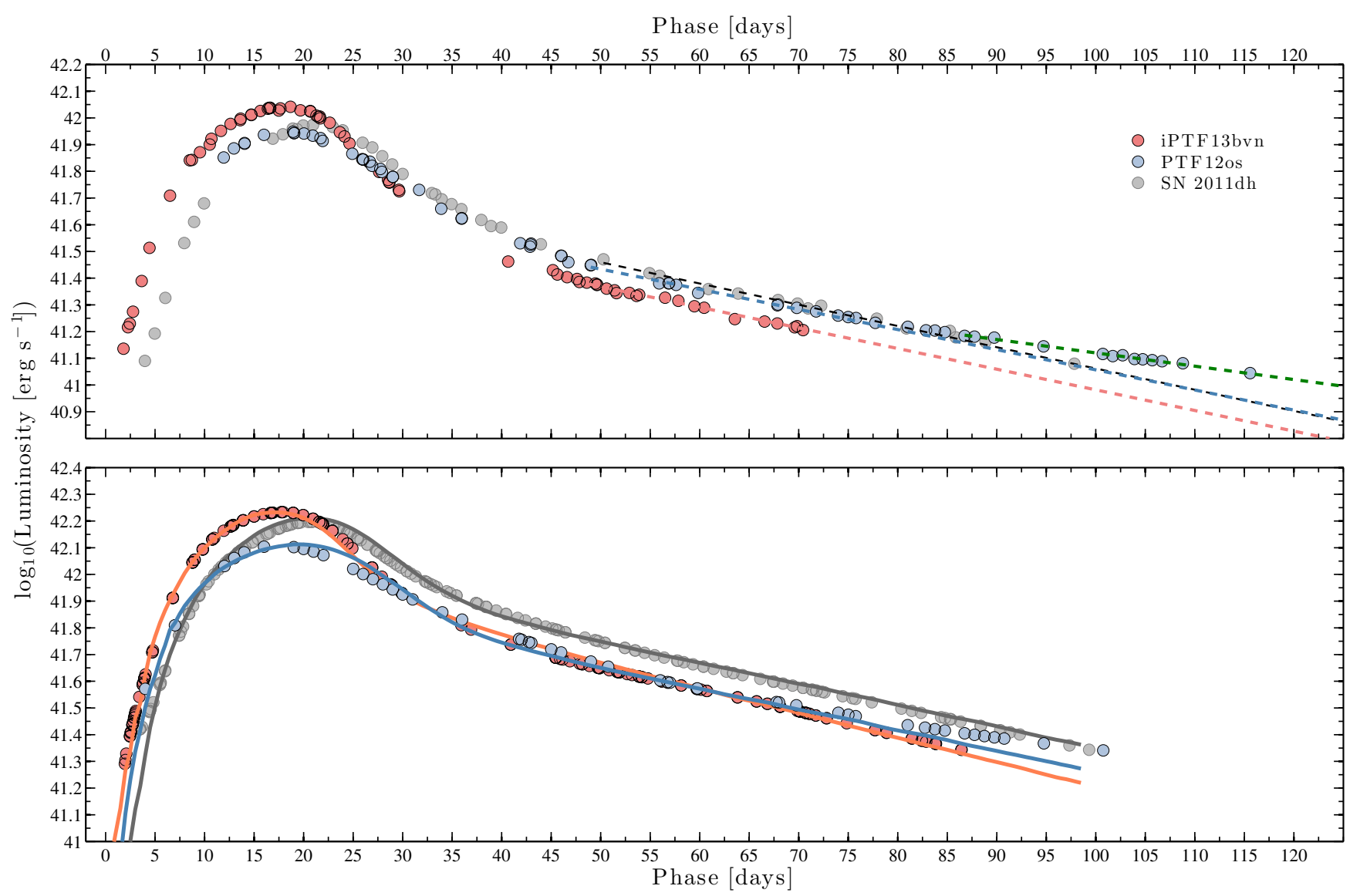

Fig. 10. Bolometric light curves of iPTF13bvn (red circles), SN 2011dh (gray circles), and PTF12os (light-blue circles). The top panel shows quasibolometric Bgriz LCs calculated as described in Sect. 6. In the top panel we also show linear fits to the LC points between $+53 \mathrm{~d}$ and $+75 \mathrm{~d}$ for the three SNe (dashed lines, PTF12os in blue, iPTF13bvn in red and SN 2011dh in black). For PTF12os an additional fit to the LC points after $+85 \mathrm{~d}$ is shown as a green dashed line, which clearly exhibits a slower decline. The bottom panel, following the same color scheme as in the top panel, illustrates the bolometric LCs of the SNe, with corrections for the UV and IR contributions based on observations of SN 2011dh applied to PTF12os and iPTF13bvn. The bottom panel also shows our best-fitting hydrodynamical models for each SN as solid lines of matching color.

differences in the multiband LCs, color properties, and quasibolometric LCs of these three SNe, the bottom line must still be that PTF12os, iPTF13bvn, and SN 2011dh are astonishingly similar photometrically.

\section{Spectroscopy}

In Sect. 6 we showed that PTF12os and iPTF13bvn are photometrically very similar. Only minor differences, both in terms of the filtered LCs and quasi-bolometric LCs, can be identified. Spectroscopically, the picture is similar, although somewhat more complex. PTF12os (Fig. A.1) and iPTF13bvn (Fig. A.2) qualitatively show the same spectral features and evolve in a similar way (see also Fig. 11). However, the spectra of iPTF13bvn generally show somewhat broader features, indicating faster expansion velocities. The spectra of PTF12os are more affected by host-galaxy contamination from the strong underlying $\mathrm{H} \alpha$ region (Sect. 5.1).

The very early spectra (until $+10 \mathrm{~d}$ ) of iPTF13bvn are dominated by broad features, indicating high expansion velocities. We can identify emission from the He I $5876 \AA$ line in the earliest spectrum of the $\mathrm{SN}$ obtained at $+2.15 \mathrm{~d}$. Its absorption minimum indicates a velocity of $17000-18000 \mathrm{~km} \mathrm{~s}^{-1}$, consistent with what was measured in the spectrum at $+2.3 \mathrm{~d}$ by Fremling et al. (2014). For PTF12os, our spectroscopic coverage starts at $+8.5 \mathrm{~d}$, and we can therefore not make a comparison of the very early expansion velocities. However, the He I $5876 \AA$ line shows a significantly slower velocity of only $7000 \mathrm{~km} \mathrm{~s}^{-1}$ compared to around $12000 \mathrm{~km} \mathrm{~s}^{-1}$ for iPTF13bvn in spectra obtained around $+8 \mathrm{~d}$.

The iPTF13bvn spectrum at $+2.15 \mathrm{~d}$ shows a significantly bluer continuum compared to the later spectra obtained at $+2.3 \mathrm{~d}$ and $+2.6 \mathrm{~d}$. Blackbody fits to these three very early-time spectra yield temperatures of $8000 \pm 500 \mathrm{~K}$ at $+2.15 \mathrm{~d}, 7000 \pm 500 \mathrm{~K}$ at $+2.3 \mathrm{~d}$, and $7500 \pm 500 \mathrm{~K}$ at $+2.6 \mathrm{~d}^{22}$ (Fig. 12). This is consistent with the cooling trend observed in the optical LCs of the SN during this phase (Sect. 6.2). Note also that a decline in temperature from around $9000 \mathrm{~K}$ to $7000 \mathrm{~K}$ is predicted to occur between $+2 \mathrm{~d}$ and $+3 \mathrm{~d}$ in the semianalytical model for the early-time photometry we used to estimate the radius of the progenitor of this SN (Sect. 6.2 and Fig. 9).

Based on spectral comparisons with other $\mathrm{SNe} \mathrm{Ib}$, iPTF13bvn has been classified as a spectroscopically normal SN Ib both in the optical and the NIR (Cao et al. 2013; Fremling et al. 2014). The emergence of He I $\lambda \lambda 5016,5876,6678$, and 7065 absorption became clear after $\sim+15 \mathrm{~d}$. The velocities of the He I $\lambda \lambda 5876,6678,7065$ lines along with the velocity of Fe II 15169, based on the respective absorption minima, were measured by Fremling et al. (2014). PTF12os shows very similar

${ }^{22}$ We note that since the spectra are actually not smooth BBs, these fits come with large systematic uncertainties. 


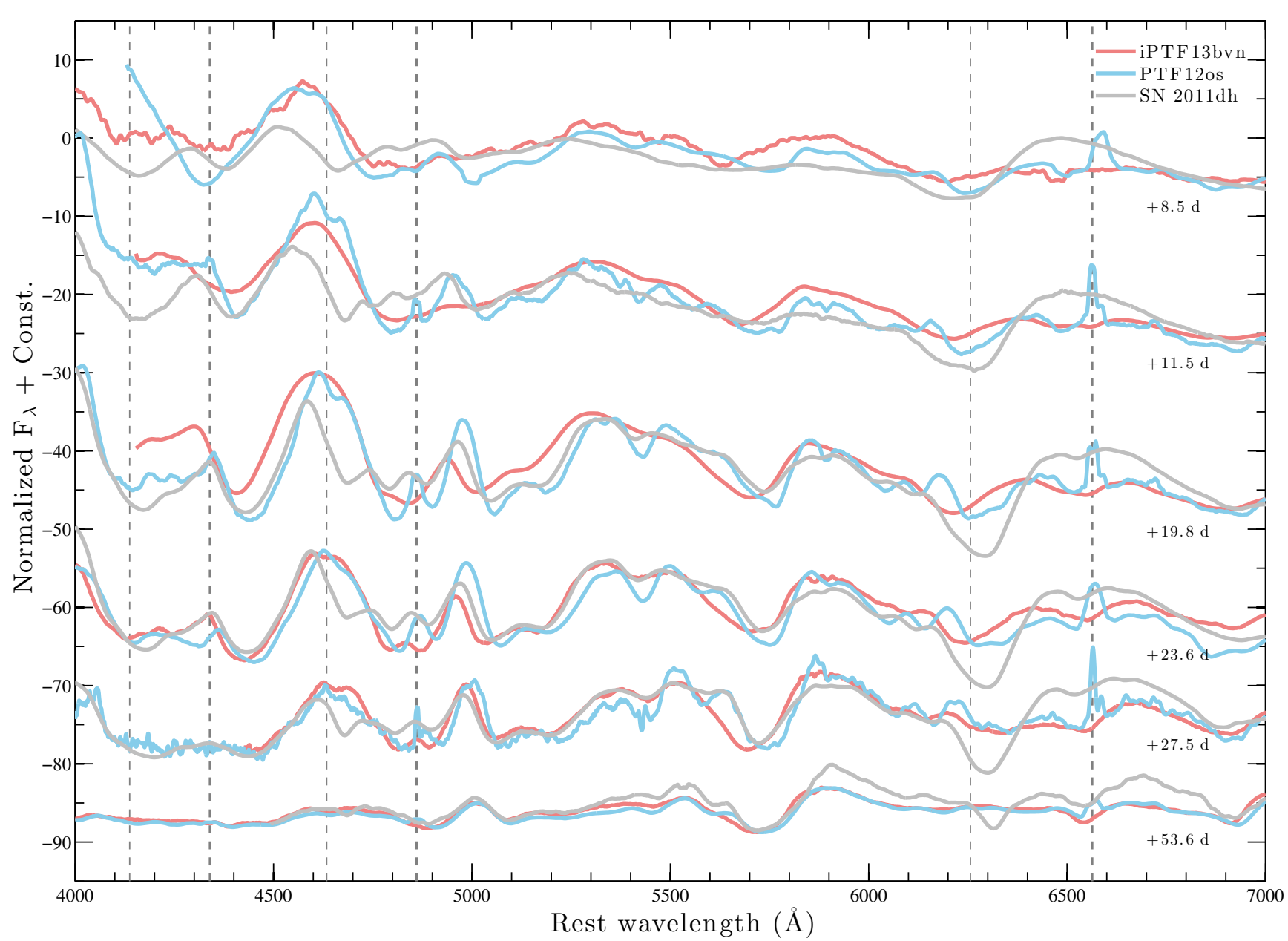

Fig. 11. Spectral comparison of PTF12os (blue), iPTF13bvn (red), and SN 2011dh (gray). The spectra have been normalized using the median and shifted by a constant for each epoch. The thick dashed lines show the rest wavelength of $\mathrm{H} \alpha, \mathrm{H} \beta$, and $\mathrm{H} \gamma$, and the thin dashed lines mark the corresponding wavelengths shifted by $14000 \mathrm{~km} \mathrm{~s}^{-1}$, which coincides very well with absorption minima in the spectra of PTF12os for all three lines. The spectra have been smoothed to improve their visibility. The date labels refer to the date of the spectral observations of PTF12os for each epoch. The spectra of the other SNe have been chosen as close as possible to these epochs.

evolution in the He I lines. In spectra taken after $+16 \mathrm{~d}$, the He I $\lambda \lambda 5016,5876,7065$ lines can be clearly identified. He I $\lambda 6678$ is difficult to distinguish in early-time spectra owing to strong host contamination, but it can be seen in our spectra taken between $+35 \mathrm{~d}$ and $+53 \mathrm{~d}$. Another minor difference between these $\mathrm{SNe}$ can be seen in the He I $\lambda 5016$ line, which is clearly seen even in our earliest spectrum of PTF12os (taken at $+8.5 \mathrm{~d}$ ), while it appears somewhat later, after $+11 \mathrm{~d}$, in iPTF13bvn. A similar effect is visible in Fe II $\lambda 5169$; after $+10 \mathrm{~d}$ this line gradually starts to appear in iPTF13bvn, while it is already there in our earliest spectrum (at $+8.5 \mathrm{~d}$ ) of PTF12os.

We have determined the He I $\lambda \lambda 5016,5876,6678,7065$ and Fe II $\lambda 5169$ velocities in all of the spectra where measurements were possible for both PTF12os and iPTF13bvn. This also included remeasuring velocities in previously published spectra, and it was done by fitting Gaussians in small regions around the absorption minima of the lines and locating the minima in the fits. In some cases, where the galaxy contamination is very strong and reasonable Gaussian fits are not possible, the centers of the absorption features were measured by manual inspection of the spectra. While we attempted velocity measurements of the HeI $\lambda 5016$ absorption, we found that it is very contaminated in most spectra of both PTF12os and iPTF13bvn; thus, we have chosen to not include this line in our comparisons.
We present the absorption-line velocity evolution of PTF12os and iPTF13bvn in Fig. 13.

As an estimate for the photospheric expansion velocity, which is employed in our hydrodynamical modeling in Sect. 8, we use the Fe II $\lambda 5169$ line (see Dessart \& Hillier 2005), which evolves from $\sim 10000 \mathrm{~km} \mathrm{~s}^{-1}$ at $+10 \mathrm{~d}$ to $5800 \mathrm{~km} \mathrm{~s}^{-1}$ at $+50 \mathrm{~d}$ in iPTF13bvn, and from $\sim 8000 \mathrm{~km} \mathrm{~s}^{-1}$ at $+10 \mathrm{~d}$ to $4500 \mathrm{~km} \mathrm{~s}^{-1}$ at $+50 \mathrm{~d}$ in PTF12os. While PTF12os shows on average slower photospheric velocities, the temporal evolution of the velocities (based on this line) is extremely similar, and the decrease in the expansion velocity is identical within our uncertainties between $+10 \mathrm{~d}$ and $+25 \mathrm{~d}$ (see the dashed lines in Fig. 13).

Similar velocity differences as for Fe II $\lambda 5169$ can be seen in the He I lines, with iPTF13bvn showing on average faster velocities compared to PTF12os. We also note that the velocity evolution of the He I lines in PTF12os appears relatively flat, with only the He I $\lambda 6678$ showing a small but statistically significant decrease in velocity during the evolution of the $\mathrm{SN}^{23}$. Note also that our spectral sequence starts at $+8.5 \mathrm{~d}$, and if the same time span were considered for iPTF13bvn, the velocity evolution would look similar. Earlier spectra of PTF12os would have

23 We also find a decline over time in the few velocity measurements that were possible for He I $\lambda 5016$ in PTF12os, but this line is strongly contaminated, as previously discussed. 


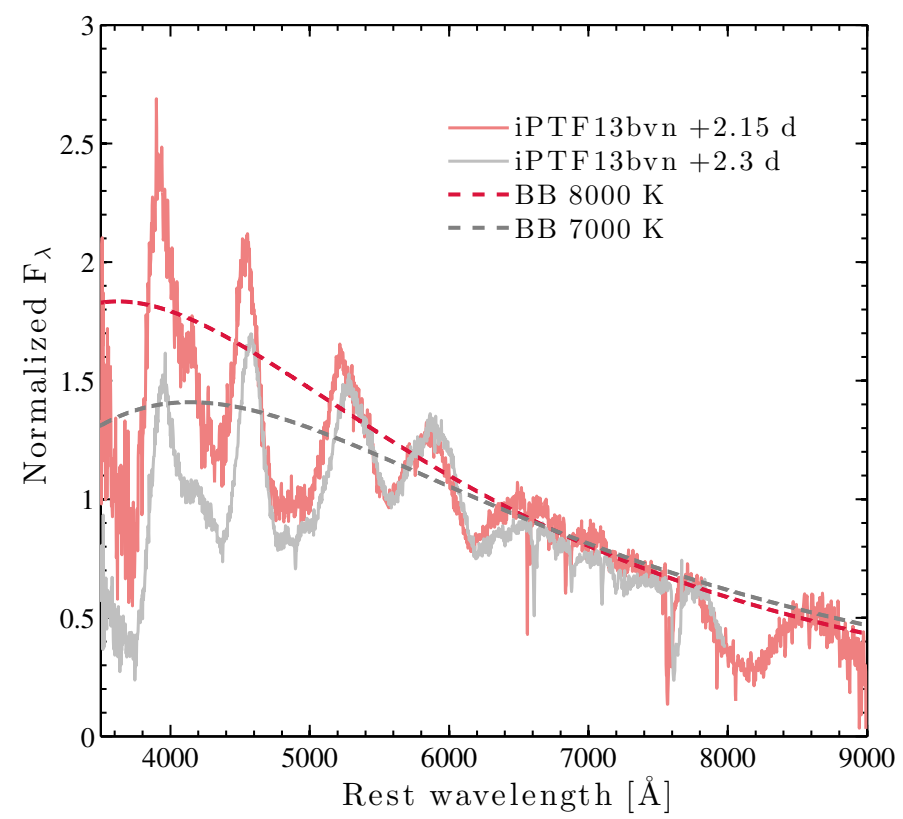

Fig. 12. Early-time spectra of iPTF13bvn obtained at $+2.15 \mathrm{~d}$ (red) and $+2.3 \mathrm{~d}$ (gray) together with blackbody fits (dashed lines, $8000 \mathrm{~K}$ in red and $7000 \mathrm{~K}$ in gray). The spectra have been normalized by their median values in a region around $6000 \AA$.

been needed to investigate any constant-velocity evolution in the He I lines, as has been suggested for some peculiar SNe IIb by Folatelli et al. (2014b).

Past $+50 \mathrm{~d},[\mathrm{O} \mathrm{I}] \lambda \lambda 5577,6300,6364$ emission lines along with the OI $\lambda 7774$ triplet start to emerge in both PTF12os and iPTF13bvn, indicating that their ejecta are gradually becoming optically thin. In our latest spectum of PTF12os, obtained at $+215 \mathrm{~d}$, weak He I signatures are still present, but the spectrum has become dominated by [OI] $\lambda \lambda 6300,6364$ and [Ca II] $\lambda \lambda 7291,7323$ emission. The Mg I] $\lambda 4571$ emission line is also present. In our late-time spectra past $+250 \mathrm{~d}$ of iPTF13bvn, the picture is very similar. The [O I] $\lambda \lambda 6300,6364$ and [Ca II] $\lambda \lambda 7291,7323$ doublets dominate. Weak Mg I] $\lambda 4571$ emission can also be identified, although there is significant noise in this region. The [Ca II] lines started to emerge after $+36 \mathrm{~d}$ in iPTF13bvn and after $+45 \mathrm{~d}$ in PTF12os.

In conclusion, the entire spectral evolution in the optical of PTF12os and iPTF13bvn is very similar, consistent with the comparable photometry (Sect. 6). We will investigate in Sect. 7.3 the late-time spectra in detail using nebular models.

\subsection{Near-infrared spectroscopy of iPTF13bvn}

In addition to the early-time NIR spectra $(+3 \mathrm{~d},+9 \mathrm{~d}$, and $+17 \mathrm{~d})$ published by Cao et al. (2013), we have obtained one later NIR spectrum at $+79 \mathrm{~d}$ with the Magellan telescope in Chile. The NIR spectral sequence, along with comparisons to SN 2011dh and SN 2008ax (Type IIb; Pastorello et al. 2008; Taubenberger et al. 2011), are shown in Fig. A.3.

In our NIR spectrum of iPTF13bvn obtained at $+79 \mathrm{~d}$, in addition to the He I $\lambda 10830$ and $\lambda 20590$ lines previously identified by Cao et al. (2013), emission features around $12000 \AA$ and $15000 \AA$ have emerged. These are likely signatures of emission from He I $\lambda 11970$ or Si I $\lambda 12000$ and a blend of Mg I $\lambda \lambda 15040$, 15048 . These identifications are based on the modeling of the NIR spectra of SN 2008ax at +130 d by Jerkstrand et al. (2015).
Figure A.3 clearly shows that the spectral evolution in the NIR regime is very similar for iPTF13bvn and SN 2008ax. In the analysis by Jerkstrand et al. (2015), Si I $\lambda 12000$ from explosively synthesized silicon is preferred over He I $\lambda 11970$ for the line emerging at $12000 \AA$. We also estimate that the He I velocities have decreased from $\sim 20000 \mathrm{~km} \mathrm{~s}^{-1}$ in the early-time spectra to $\sim 15000 \mathrm{~km} \mathrm{~s}^{-1}$ at $+79 \mathrm{~d}$, measured from the absorption minimum of the $\mathrm{He} \mathrm{I} \lambda 10830$ line.

Early-time NIR spectra of SN 2011dh show strong signatures of hydrogen, as seen clearly by the presence of emission features at Paschen $\beta$ and Paschen $\delta$ at $12820 \AA$ and $10050 \AA$ (Fig. A.3). Paschen $\gamma$ is blended with strong emission of He I $\lambda 10830$. Note also that we do not have data for SN $2011 \mathrm{dh}$ in the region of Paschen $\alpha$ owing to telluric absorption.

For iPTF13bvn, no clear signatures of hydrogen can be seen. However, compared to an estimate of the continuum, found by heavily smoothing the spectra, there is a possible excess in both the $+3 \mathrm{~d}$ and $+9 \mathrm{~d}$ spectra around the Paschen $\beta$ line (Fig. A.3). Furthermore, the NIR spectrum of the Type IIb SN 2008ax $(+10 \mathrm{~d}$; Taubenberger et al. 2011) is very similar to the spectrum of iPTF13bvn at $+9 \mathrm{~d}$, especially in the regions around the Paschen lines. This indicates that the observed NIR spectra of iPTF13bvn do not exclude the possibility of the presence of a small amount of hydrogen at early times. The Paschen $\alpha$ line falls in a region with strong telluric absorption in all our NIR spectra of iPTF13bvn, so we are not able to draw conclusions about the presence of potential broad Paschen $\alpha$ emission. We discuss the presence of hydrogen in the optical spectra of both iPTF13bvn and PTF12os in the following section.

\subsection{Hydrogen in early spectra}

The similarity of PTF12os to iPTF13bvn would logically lead to a Type Ib classification for PTF12os. However, in our earlytime spectra of PTF12os we can identify likely signatures of $\mathrm{H} \alpha$, $\mathrm{H} \beta$, and $\mathrm{H} \gamma$ in absorption at $\sim 14000 \mathrm{~km} \mathrm{~s}^{-1}$. This is illustrated by the dashed green lines in Fig. A.1 and also by the dashed lines in Fig. 11, where we illustrate a comparison of early-time spectra of PTF12os, iPTF13bvn, and SN 2011dh. The spectrum of PTF12os at $+19 \mathrm{~d}$ is very similar to spectra of the Type IIb SN 2011dh obtained at similar epochs (see also the bottom panel of Fig. A.1).

In iPTF13bvn, no signatures of $\mathrm{H} \beta$ or $\mathrm{H} \gamma$ can be identified, but the spectra between $+10 \mathrm{~d}$ and $+25 \mathrm{~d}$ could still be consistent with the presence of $\mathrm{H} \alpha$ since the spectral evolution is very similar to that of PTF12os, especially in this region; the absorption feature around $6300 \AA$ is comparable in strength and it disappears after $\sim+25 \mathrm{~d}$ in both $\mathrm{SNe}$ (see Fig. 14). For PTF12os, based on the comparison to SN 2011dh, it appears very likely that this feature is due to hydrogen, and thus it could be argued that it should be the case also for iPTF13bvn. The early-time NIR spectra of iPTF13bvn (Fig. A.3) are also very similar to NIR spectra of the Type IIb SN 2008ax around the Paschen lines, so these data do not exclude the presence of a hydrogen envelope, as already discussed in Sect. 7.1. The lack of $\mathrm{H} \beta$ (and $\mathrm{H} \gamma$ ) signatures in iPTF13bvn may possibly be explained by the higher expansion velocities, making the lines more difficult to identify.

In spectra of SN 2011dh, a clear P-Cygni signature from $\mathrm{H} \alpha$ at $\sim 6300 \AA$ is seen in spectra obtained after $+4 \mathrm{~d}$. The strength of this feature compared to the continuum reached a maximum at around $+25 \mathrm{~d}$, whereafter it gradually weakened and disappeared after $\sim+90 \mathrm{~d}$, leading to the Type IIb classification. Spectral signatures of $\mathrm{H} \beta$ and $\mathrm{H} \gamma$ with similar behaviors could also be 


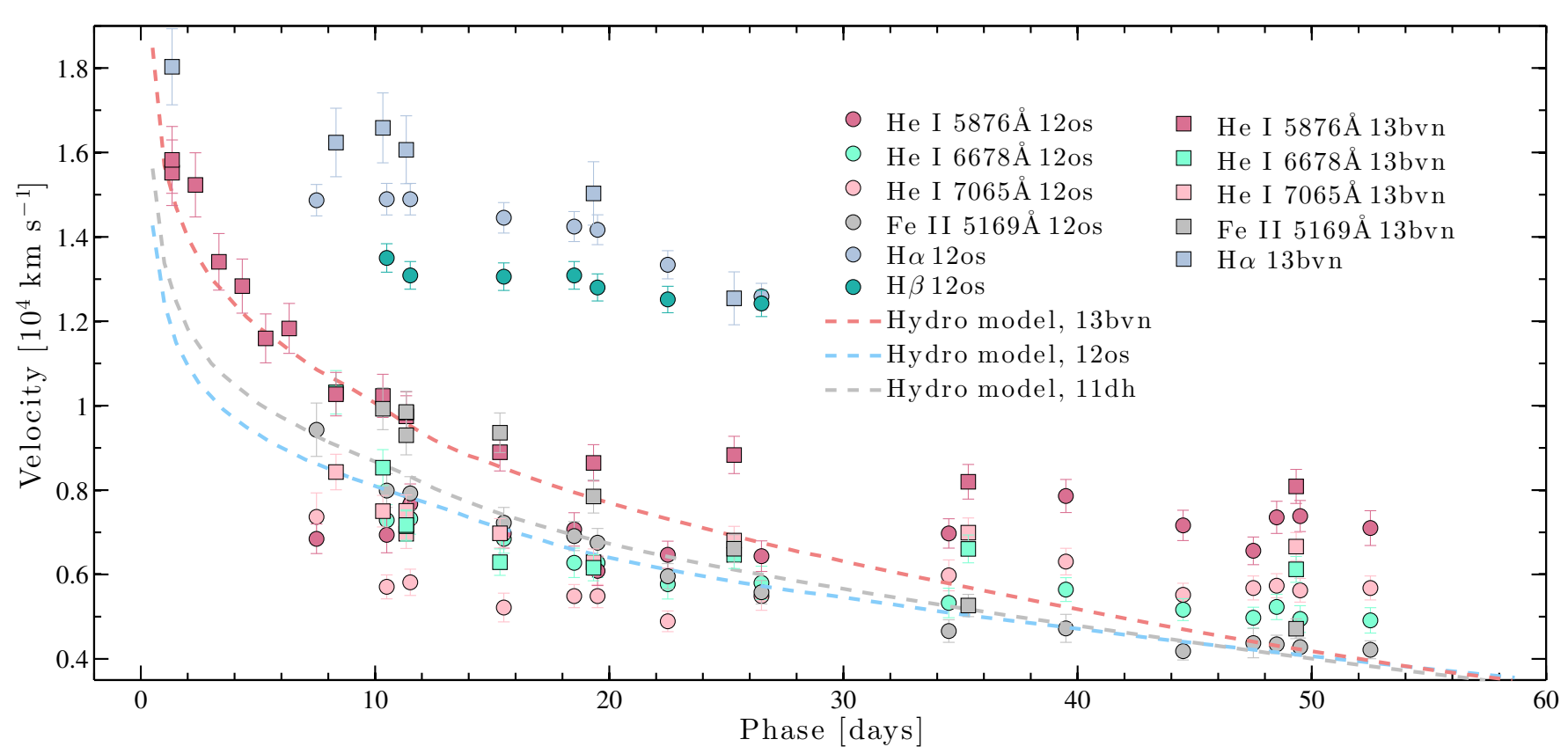

Fig. 13. Absorption minimum velocity measurements for a selection of spectral lines in iPTF13bvn (squares) and PTF12os (circles). The velocity of He I $\lambda 5876$ is shown in red, He I $\lambda 6678$ in cyan, and He I $\lambda 7065$ in pink. The Fe II $\lambda 5169$ absorption minimum velocities are shown in gray. We also indicate the estimated $\mathrm{H} \alpha$ (blue) and $\mathrm{H} \beta$ (teal) velocities for both PTF12os and iPTF13bvn. The dashed lines show the photospheric velocity evolution of our best-fitting hydrodynamical models for SN 2011dh (gray), PTF12os (blue), and iPTF13bvn (red).

identified. Monte-Carlo-based spectral modeling was used by Ergon et al. (2014) to estimate the mass of the hydrogen envelope of the progenitor of SN $2011 \mathrm{dh}$ as $0.01-0.04 M_{\odot}$, in agreement with the non-local thermodynamic equilibrium modeling by Arcavi et al. (2011), who estimate the hydrogen mass in the envelope of SN 2011dh to be $0.024 M_{\odot}$.

Based on the sequence shown in Fig. 11, it is clear that much stronger hydrogen features developed in SN 2011dh compared with both PTF12os and iPTF13bvn. The absorption feature at $6300 \AA$ is there at $+8.5 \mathrm{~d}$ in PTF12os (our earliest spectrum of this SN), and it becomes clear at around $+10 \mathrm{~d}$ in iPTF13bvn. In both $\mathrm{SNe}$, the absorption disappears after around $+25 \mathrm{~d}$ (compared to $\sim+90 \mathrm{~d}$ in SN 2011dh). One interpretation of this spectral behavior is that we are seeing the signature of a hydrogen envelope around the progenitor, just as what is seen in SNe IIb such as SN 2011dh. If we assume that this absorption is caused by the $\mathrm{H} \alpha$ line, we can estimate the expansion velocity of the hydrogen envelope by measuring the position of the absorption minimum using Gaussian fits. We find that $\mathrm{H} \alpha$ in iPTF13bvn would be at $\sim 16000 \mathrm{~km} \mathrm{~s}^{-1}$ at $+10 \mathrm{~d}$, about $2000 \mathrm{~km} \mathrm{~s}^{-1}$ faster compared to PTF12os (left panel of Fig. 14 and also Fig. 13 for the velocity measurements). We have also investigated the region where we would expect $\mathrm{H} \beta$ (right panel of Fig. 14), and we find that for PTF12os there is absorption at $\sim 13000 \mathrm{~km} \mathrm{~s}^{-1}$ in several of the early spectra before $+25 \mathrm{~d}$. The velocity evolutions of the $\mathrm{H} \alpha$ and $\mathrm{H} \beta$ lines are consistent with what was observed in SN 2011dh, except that PTF12os and especially iPTF13bvn exhibit higher velocities. Faster expansion velocities are expected, since the mass of the hydrogen envelopes appear to be smaller in PTF12os and iPTF13bvn compared with SN 2011dh, given the weaker spectral signatures.

In the modeling effort by Hachinger et al. (2012), it was found that as little as $0.024 M_{\odot}$ of hydrogen in the envelope is enough for $\mathrm{H} \beta$ (and a strong signal from $\mathrm{H} \alpha$ ) to show up in the spectra of a $\mathrm{SN} \mathrm{IIb/Ib;} \mathrm{thus,} \mathrm{a} \mathrm{hydrogen} \mathrm{envelope} \mathrm{mass}$ upper limit of $\lesssim 0.02 M_{\odot}$ is a reasonable estimate for PTF12os (but probably rather high, given the very similar estimate for SN 2011dh, which shows much more long-lasting hydrogen signatures). For iPTF13bvn the hydrogen mass is likely lower, since we see no signs of $\mathrm{H} \beta$. However, the higher velocities in combination with the fact that the $\mathrm{H} \alpha$ absorption disappears at the same time as for PTF12os could be seen as an argument for at least a comparable hydrogen mass, since the emission from the same amount of hydrogen as in PTF12os should disappear earlier for a higher expansion velocity, assuming that the other physical parameters of the explosion are similar. Detailed physical modeling of the spectra of iPTF13bvn is needed to put a realistic upper limit on the hydrogen mass, especially since the modeling by Dessart et al. (2011) found that even with an envelope mass as low as $0.001 M_{\odot}, \mathrm{H} \alpha$ will still be present at $+15 \mathrm{~d}$ - a very small amount of hydrogen is needed to produce a weak spectral signature at early times.

In the spectral analysis of SE SNe by Parrent et al. (2016), it is also suggested that several other $\mathrm{SNe} \mathrm{Ib}$ in the literature besides iPTF13bvn show weak signatures of $\mathrm{H} \alpha$ in absorption at early times. This conclusion is also consistent with predictions from binary evolution (e.g., Yoon 2015), where it is found that a small amount of hydrogen can remain in some cases for models otherwise tuned to produce $\mathrm{SNe} \mathrm{Ib}$.

Milisavljevic et al. (2013b) suggested that the Type IIb vs. Ib classification is time dependent. If the first spectra of PTF12os and iPTF13bvn had been obtained after $+25 \mathrm{~d}$, these objects would simply have been classified as $\mathrm{SNe} \mathrm{Ib}$. With knowledge of the early-time spectral evolution they instead appear very similar to the Type IIb SN 2011dh, although likely with a smaller amount of hydrogen. In the Type IIb SN 2008ax, the hydrogen signatures disappeared after $\sim+45 \mathrm{~d}$. There could indeed be a continuum between $\mathrm{SNe}$ IIb and $\mathrm{SNe} \mathrm{Ib}$, with $\mathrm{SE} \mathrm{SNe}$ showing varying degrees of stripping of the progenitor. This would be in contrast with the previously suggested bimodal picture 


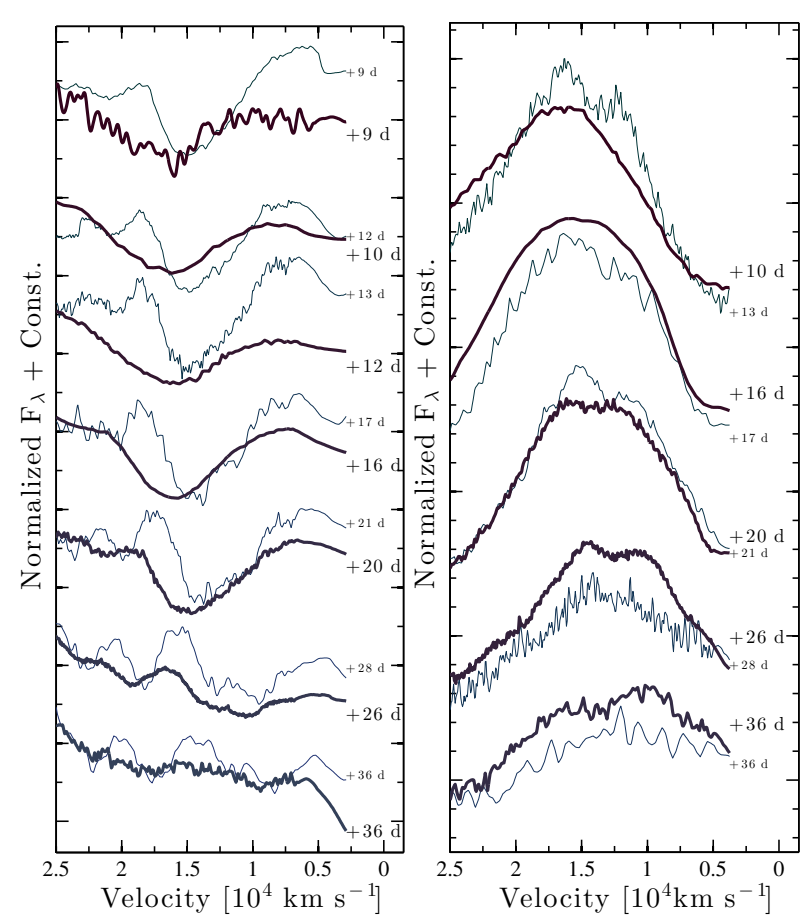

Fig. 14. Spectral evolution in velocity space around $\mathrm{H} \alpha$ (left panel) and $\mathrm{H} \beta$ (right panel) of iPTF13bvn (thick black line) and PTF12os (thin dark blue line).

in which $\mathrm{SNe} \mathrm{IIb}$ and $\mathrm{SNe} \mathrm{Ib}$ come from different progenitor channels.

However, recently Liu et al. (2016) analyzed a large sample of SN IIb and SN Ib spectra, claiming that the strength of the $\mathrm{H} \alpha$ absorption, as measured using the pseudoequivalent width (pEW; Blondin et al. 2011; Silverman et al. 2012), shows a bimodal distribution with a clear separation between $\mathrm{SNe}$ IIb and $\mathrm{SNe} \mathrm{Ib}$ at all epochs, with $\mathrm{SNe}$ IIb showing stronger absorption. Liu et al. (2016) also find that iPTF13bvn seems to be an outlier; in terms of the strength of the $\mathrm{H} \alpha$ absorption it is comparable to the $\mathrm{SNe}$ IIb that exhibit the weakest hydrogen signatures. In Fig. 14 it is clear that the strength of $\mathrm{H} \alpha$ is also similar for PTF12os at all epochs. Thus, it might indeed be that iPTF13bvn is a special case, and should perhaps be considered a SN IIb. More early-time spectra of SNe IIb/Ib, with well-determined explosion dates from early photometry, are needed to further investigate the nature of the hydrogen envelopes of their progenitors.

\subsection{Nebular spectroscopy and oxygen-mass constraints}

Figure 15 shows a comparison of late-time spectra of PTF12os (around $+215 \mathrm{~d}$ ) and iPTF13bvn (at $+251 \mathrm{~d},+345 \mathrm{~d}$, and $+346 \mathrm{~d}$ ), along with fits based on the nebular models by Jerkstrand et al. (2015) for SNe IIb. The predicted luminosity of the $[\mathrm{OI}] \lambda \lambda 6300,6364$ doublet found by Jerkstrand et al. (2015) was previously used by Fremling et al. (2014) to constrain the ZAMS mass of iPTF13bvn. This was based on nebular $r$-band photometry under the assumption that the $r$-band flux is dominated by the [O I] $\lambda \lambda 6300,6364$ doublet. An upper limit of $\sim 17 M_{\odot}$ on the ZAMS mass of iPTF13bvn was derived. Since then, Kuncarayakti et al. (2015) have arrived at a similar limit on the ZAMS mass using independent nebular spectroscopy obtained at around $+300 \mathrm{~d}$.

We find that our spectra obtained between $+251 \mathrm{~d}$ and $+346 \mathrm{~d}$ of iPTF13bvn give even stricter constraints. The same model that was found to be optimal for SN 2011 dh (model 12C 24 in Jerkstrand et al. 2015) produces good fits to the observed spectra of iPTF13bvn at these epochs, and especially to the [OI] $\lambda \lambda 6300,6364$ doublet, is very well reproduced (see the middle and bottom panels of Fig. 15). In this model the oxygen mass is $\sim 0.3 M_{\odot}$, which translates into a ZAMS mass of $12 M_{\odot}$. The $\mathrm{Mg}$ I] $\lambda 4571$ line is also present in our spectrum at $+251 \mathrm{~d}$, and the $12 M_{\odot}$ model roughly reproduces this line as well. To produce the model fits for iPTF13bvn we have flux-calibrated our nebular spectra using the extinction-corrected $r$-band flux, scaled the model by the nickel mass derived for iPTF13bvn $\left(0.072 M_{\odot}\right.$; see Sect. 8), and scaled the model in time to match the epoch of our spectra according to the prescriptions of Jerkstrand et al. (2015).

We note that in the binary model for iPTF13bvn by Bersten et al. (2014), a relatively low oxygen mass is predicted, even though an initial mass of $20 M_{\odot}$ for the SN progenitor star is assumed. In a single-star setting, an oxygen mass around $2 M_{\odot}$ would be expected for such a star. However, in the model by Bersten et al. (2014), the mass transfer to the companion starts during core hydrogen burning, which results in a reduced oxygen mass in the core of the SN progenitor. Thus, our ZAMS estimate of $12 M_{\odot}$ should be treated with some caution, since the models by Jerkstrand et al. (2015) are based on the singlestar nucleosynthesis models of Woosley \& Heger (2007), and binary models can arrive at a He core with similar properties to our best-fitting model at the time of explosion for larger initial masses (see, e.g., Eldridge et al. 2015; Yoon et al. 2010). On the other hand, Eldridge \& Maund (2016) have recently constructed a binary model for the iPTF13bvn system based on both pre-explosion and late-time $(+740 \mathrm{~d})$ HST data, where the progenitor mass of the $\mathrm{SN}$ is constrained to $10-12 M_{\odot}$, which is in excellent agreement with the result from our nebular modeling.

Following the same procedure as above, we have attempted to fit the same nebular model to PTF12os as for iPTF13bvn, but now scaled to the nickel mass for PTF12os $\left(0.063 M_{\odot}\right.$; see Sect. 8). However, this model does not produce sufficiently strong emission lines for this SN (see the gray line in the top panel of Fig. 15). A model with a ZAMS mass of $13 M_{\odot}$ (model $13 \mathrm{G}$ of Jerkstrand et al. 2015) and otherwise identical parameters as the model used for iPTF13bvn gives decent matches to both the quasicontinuum and the emission lines of PTF12os (gray line in the top panel of Fig. 15). On the other hand, the [O I] $\lambda \lambda 6300$, 6364 doublet is still too weak.

In Sect. 6.3 we found that while the quasibolometric LC peak is lower for PTF12os, the LC is declining slower compared to SN 2011dh past $+80 \mathrm{~d}$, and at $+105 \mathrm{~d}$ the quasibolometric luminosity of both $\mathrm{SNe}$ is almost the same. At later times we lack coverage in the $B$ band; however, the fluxes in the gri bands are almost the same in PTF12os at $+215 \mathrm{~d}$ as in SN 2011dh. The slower decline of PTF12os implies more-efficient $\gamma$-ray trapping compared to SN 2011dh (and iPTF13bvn). If the nebular model were adjusted accordingly, the lines should be stronger for a lower nickel mass at late times.

One way to achieve more-efficient $\gamma$-ray trapping is to simply increase the ZAMS mass of the model, resulting in increased ejecta and oxygen masses. In Fig. 15 we also show a model for a star with ZAMS mass of $17 M_{\odot}$ (model 17A of Jerkstrand et al. 2015). The [O I] $\lambda \lambda 6300,6364$ lines are significantly too strong in this model, and the ratio between this doublet and the

\footnotetext{
${ }^{24}$ Model $12 \mathrm{C}$ has strong mixing, local positron trapping, and no molecular cooling, and it assumes that there is dust formation after $+200 \mathrm{~d}$ (for further details, see Jerkstrand et al. 2015).
} 


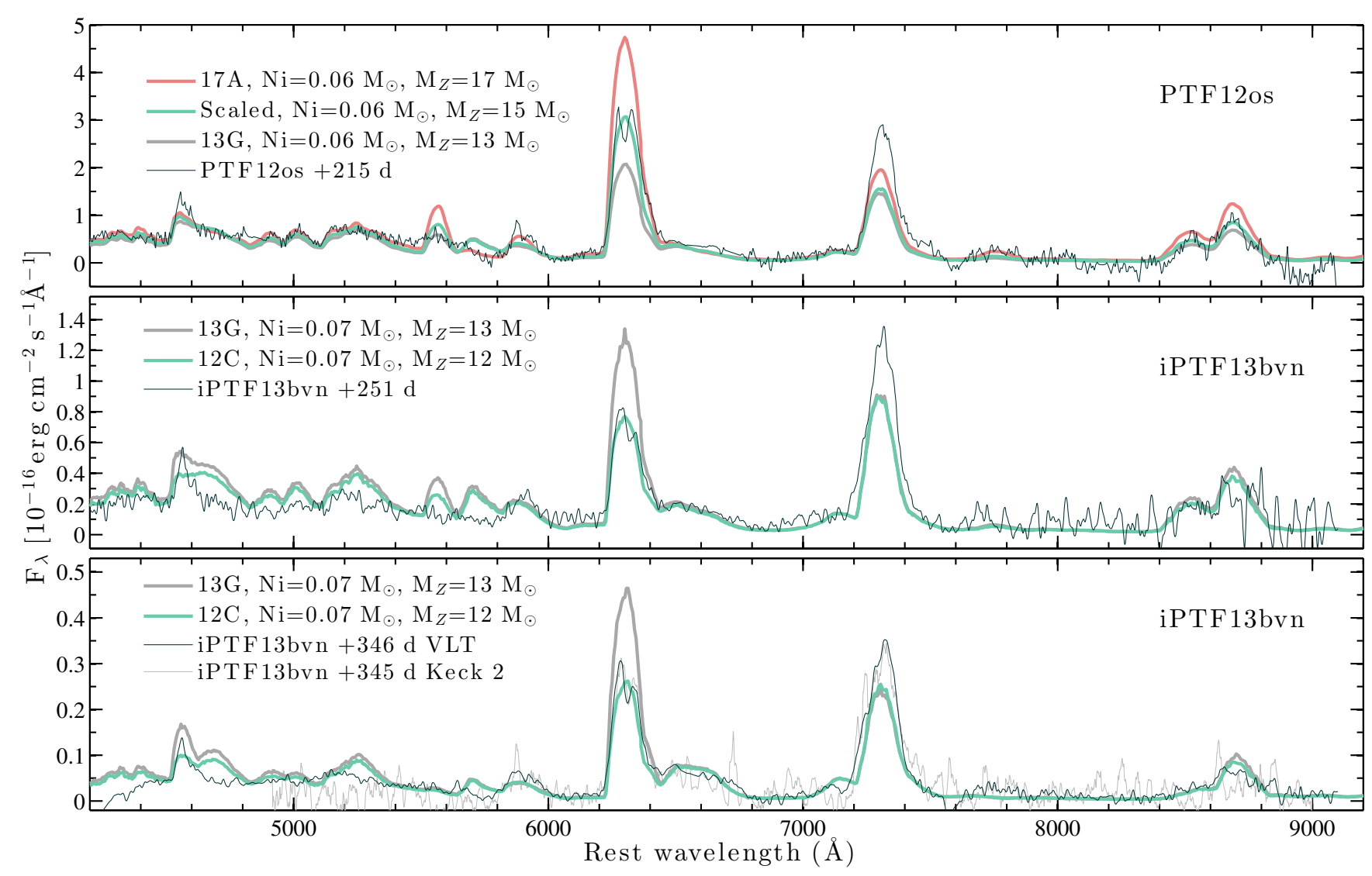

Fig. 15. Nebular spectra of PTF12os (top panel) and iPTF13bvn (middle and bottom panels) compared with nebular models. For PTF12os we show a flux-calibrated nebular spectrum (black line) obtained at $+215 \mathrm{~d}$. Nebular models with $M_{\text {ZAMS }}=13 M_{\odot}$ (gray line) and $M_{\text {ZAMS }}=17 M_{\odot}$ (red line) are also shown, along with a model scaled in oxygen mass to represent a star with $M_{\text {ZAMS }}=15 M_{\odot}$ (green line). The observed spectrum is consistent with the model representing $M_{\text {ZAMS }}=15 M_{\odot}$. For iPTF13bvn we show three flux-calibrated spectra, one observed at $+251 \mathrm{~d}$ (black line, middle panel), one at $+345 \mathrm{~d}$ (gray line, bottom panel) and one at $+346 \mathrm{~d}$ (black line, bottom panel). These spectra are consistent with a model having $M_{\text {ZAMS }}=12 M_{\odot}$ (green lines). All models have been scaled to the epochs of the spectra according to the prescriptions of Jerkstrand et al. (2015).

[Ca II] $\lambda \lambda 7291,7323$ doublet is significantly off compared to the observation. Thus, it appears that a ZAMS mass of $17 M_{\odot}$ is too large for PTF12os. If we interpolate the model spectra as a function of the oxygen mass (using models $12 \mathrm{C}, 13 \mathrm{G}$, and $17 \mathrm{~A}$ of Jerkstrand et al. 2015), we find that the [O I] $\lambda \lambda 6300,6364$ lines are very well reproduced by a model with an oxygen mass of $\sim 0.8 M_{\odot}$ or a ZAMS mass of $15 M_{\odot}$ (see the green line in the top panel of Fig. 15). We note that the He core mass of a $15 M_{\odot}$ model is still roughly consistent with the limits on the ejecta mass derived from our hydrodynamical model of PTF12os (Sect. 8).

In terms of luminosity, the [O I] $\lambda \lambda 6300,6364$ doublet luminosity normalized by the energy released by the decaying nickel mass $\left(0.063 M_{\odot}\right)$ is also significantly lower at both $+140 \mathrm{~d}$ and $+215 \mathrm{~d}$ in PTF12os than what is predicted for the model with a ZAMS mass of $17 M_{\odot}$. We measure $L_{\text {Norm }} \approx 0.017$ at $+140 \mathrm{~d}$ and $L_{\text {Norm }} \approx 0.026$ at $+215 \mathrm{~d}$, following the description of Jerkstrand et al. (2015). These numbers are roughly comparable to what has been measured for SN 2008ax and SN 1993J at similar epochs. SN 2008ax is well matched by a model with a ZAMS mass of $13 M_{\odot}$.

We have also compared the models presented above to nebular models of iPTF13bvn (for +346d) and PTF12os (for +215 d) calculated with the spectral code by Mazzali et al. (2007, 2010). The results from these models are also consistent with low-mass ZAMS stars $\left(<17 M_{\odot}\right)$ as the progenitors to these SNe. The best-fitting model of PTF12os has an oxygen mass of $0.73 M_{\odot}$, and the model of iPTF13bvn has an oxygen mass of $0.2 M_{\odot}$.

In conclusion, we arrive at an upper limit of $0.3 M_{\odot}$ for the oxygen mass of iPTF13bvn and $0.8 M_{\odot}$ for PTF12os. This translates to an upper limit on the ZAMS mass of $12 M_{\odot}$ for iPTF13bvn and $15 M_{\odot}$ for PTF12os for single-star ZAMS models.

\section{Hydrodynamical light-curve modeling and progenitor constraints}

Following our earlier work on iPTF13bvn (Fremling et al. 2014), we have constructed a similar hydrodynamical model for PTF12os. As input data we use the multiband $U V M 2^{25}, U B$, and griz LCs of PTF12os, a rough explosion date estimate based on an exponential fit to the early-time $r$-band LC as an initial guess, and the measured photospheric velocities (Sect. 7), together with a grid of SN models constructed with the hydrodynamical code HYDE (Ergon et al. 2015). This is a one-dimensional code based on flux-limited diffusion, and it follows the framework outlined by Falk \& Arnett (1977).

25 We choose to use only the $U V M 2$ band to estimate the UV contributions, since this Swift/UVOT band is least affected by leakage from redder wavelengths (see Ergon et al. 2014, for a discussion). 
To model PTF12os we have generated a new model grid, based on bare He cores evolved until the verge of core collapse using MESA (Paxton et al. 2010), and exploded with the latest version of the HYDE code. To allow direct comparisons we have also redone the fitting of iPTF13bvn and SN 2011dh to this same model grid.

For the IR and UV regions, at epochs where we lack coverage in our datasets on PTF12os and iPTF13bvn, we use bolometric corrections derived from the bolometric LC of SN 2011dh. When we perform the fits to our model grid, we place equal weights on the diffusion phase of the LC $(+1 \mathrm{~d}$ to $+40 \mathrm{~d})$, the early tail of the LC $(+40 \mathrm{~d}$ to $+100 \mathrm{~d})$, and the photospheric velocity measurements (which are fitted up to $+40 \mathrm{~d}$ only). From the best-fitting models, shown in the bottom panel of Fig. 10 along with the estimated bolometric LCs, we derive the explosion parameters for PTF12os, iPTF13bvn, and SN 2011dh shown in Table 2. The results on both iPTF13bvn and SN 2011dh are consistent with previous findings (Fremling et al. 2014; Ergon et al. 2014).

For PTF12os we derive a compact He core of $3.25_{-0.56}^{+0.77} M_{\odot}$, which corresponds to an ejecta mass of $1.9_{-0.56}^{+0.77} M_{\odot}$ assuming that a $1.4 M_{\odot}$ compact remnant remained at the center of the explosion. We also find that $0.063_{-0.011}^{+0.020} M_{\odot}$ of ${ }^{56} \mathrm{Ni}$ was synthesized in the explosion which had a total kinetic energy of $0.54_{-0.25}^{+0.41} \times 10^{51} \mathrm{erg}$, and that the nickel needs to be strongly mixed. If we let the explosion time vary by $\pm 2.5 \mathrm{~d}$ around the initial guess, we find the explosion epoch of the best-fitting model to be $t_{\exp }=2455933.0$ days in JD, or $2012 \mathrm{Jan}$. 6.5 in UT. The errors reported here and in Table 2 are propagated from the uncertainties in the observed quantities, but we do not take the degeneracy between the explosion parameters into account (e.g., ejecta mass vs. explosion energy) when determining the errors.

Our models for all three $\mathrm{SNe}$ are qualitatively well fitted to the observed datasets, including both the overall LCs and the photospheric velocities (see Fig. 13 for the photosphericvelocity fits for PTF12os and iPTF13bvn). In agreement with the discussion of the quasibolometric LCs in Sect. 6, we find that iPTF13bvn and SN 2011dh has the highest nickel masses $\left(0.072\right.$ and $\left.0.075 M_{\odot}\right)$, followed by PTF12os $\left(0.063 M_{\odot}\right)$.

In terms of the ejecta mass, we find that PTF12os has the highest upper limit on the ejecta mass at $2.6 M_{\odot}$ and SN $2011 \mathrm{dh}$ the lowest at $2.4 M_{\odot}$ (see Table 2). However, within the uncertainties the differences are not significant. We do not find a clearly lower ejecta mass for iPTF13bvn, although the width of the bolometric LC peak is narrower. This is because the expansion velocities are higher: a higher photospheric expansion velocity leads to a faster evolution of the LC around peak for the same ejecta mass. There is also some indication that iPTF13bvn could have a higher explosion energy compared to SN 2011dh and PTF12os, but within the errors the difference is not significant. Finally, past $+70 \mathrm{~d}$, the observed bolometric LC of PTF12os gradually starts to show a somewhat slower decline than what is seen in our best-fitting model. Models with slower late-time declines (higher ejecta mass, or lower energy, or lower velocities) fit less well to the rest of the data we are fitting. Similarly as to what we found when analyzing the quasibolometric LCs in Sect. 6.3, this again indicates that the gamma-ray trapping could be more efficient in PTF12os compared to iPTF13bvn and SN 2011dh.

\section{Discussion, summary, and conclusions}

Our observational data on PTF12os and iPTF13bvn show that these two SNe are SE SNe, with explosion parameters typical
Table 2. Explosion parameters from HYDE.

\begin{tabular}{llllc}
\hline \hline SN & $M_{\mathrm{He}}\left(M_{\odot}\right)$ & $E\left(10^{51} \mathrm{erg}\right)$ & $M_{\mathrm{Ni}}\left(M_{\odot}\right)$ & $\mathrm{Mix}_{\mathrm{Ni}}$ \\
\hline SN 2011dh & $3.31_{-0.57}^{+0.53}$ & $0.64_{-0.31}^{+0.38}$ & $0.075_{-0.020}^{+0.028}$ & $1.05_{-0.00}^{+0.08}$ \\
PTF12os & $3.25_{-0.56}^{+0.77}$ & $0.54_{-0.25}^{+0.41}$ & $0.063_{-0.011}^{+0.020}$ & $1.55_{-0.16}^{+0.07}$ \\
iPTF13bvn & $3.38_{-0.50}^{+0.57}$ & $0.94_{-0.43}^{+0.63}$ & $0.072_{-0.016}^{+0.024}$ & $1.28_{-0.00}^{+0.46}$ \\
\hline
\end{tabular}

Notes. He core mass, explosion energy, and mass and distribution of ${ }^{56} \mathrm{Ni}$ derived from the bolometric light curves of PTF12os and iPTF13bvn presented in this work and from the bolometric LC of SN 2011dh by Ergon et al. (2014). The errors include the uncertainties in the distance, extinction, and photospheric velocities, but do not take into account the degeneracy between the explosion parameters in the solutions.

of Type IIb or Ib SNe. Compared to the Type IIb SN 2011dh, the multiband light curves, the bolometric light curves, and the spectral evolution of these three $\mathrm{SNe}$ are qualitatively very similar. This is reflected in the output when we fit these SNe to our hydrodynamical model grid (Sect. 8).

We classify PTF12os as a SN IIb, with only a small part $\left(\lesssim 0.02 M_{\odot}\right)$ of the hydrogen envelope of the progenitor star remaining prior to the explosion. This conclusion is based on the presence of absorption from several of the Balmer lines in our spectral sequence (Fig. A.1), and on the temporal evolution of the $\mathrm{H} \alpha$ line. Compared to the sample studied by Liu et al. (2016), the velocity of the $\mathrm{H} \alpha$ absorption in PTF12os is on the higher end, whereas the pEW of this absorption feature is on the lower end, of the SN IIb sample. Compared to typical SNe IIb such as SN 2011dh, the signature from hydrogen is weaker in the spectra of PTF12os (Fig. 11), and it disappeared quite early at around $+25 \mathrm{~d}$. Signatures of $\mathrm{H} \alpha$ lingered in the spectra of SN 2011dh until approximately $+90 \mathrm{~d}$. All of these measurements indicate a lower mass of hydrogen in the envelope surrounding the progenitor of PTF12os compared to the progenitor of SN 2011dh. However, detailed modeling is needed to quantify the hydrogen envelope mass.

For iPTF13bvn the pEW of the $\mathrm{H} \alpha$ absorption is close to that of PTF12os and it evolves in a similar way. This means that iPTF13bvn is among the $\mathrm{SNe} \mathrm{Ib}$ with the largest $\mathrm{pEW}$ values in the sample studied by Liu et al. (2016), comparable in pEW to the lower end of the SN IIb sample where PTF12os is also found. It is not completely unreasonable to assume that a similar amount of hydrogen was present in an envelope surrounding the progenitor of iPTF13bvn as for the progenitor of PTF12os.

A similar mass of hydrogen as in PTF12os for iPTF13bvn would likely result in a radius of the progenitor prior to explosion that is significantly larger than the value of just a few solar radii reported by $\mathrm{Cao}$ et al. (2013) by fitting the equations of Piro \& Nakar (2013) to the early $r$-band LC. When we fitted the temperature, the early bolometric LC, and the early gri LCs of iPTF13bvn simultaneously using the same semianalytical framework, with the explosion parameters from our hydrodynamical model of iPTF13bvn as input, this resulted in a best-fitting radius for the progenitor on the order of $10 R_{\odot}$. Hydrodynamical modeling by Bersten et al. (2014) is also consistent with a significantly larger radius for iPTF13bvn. The binary models by Yoon et al. (2010) show that it is likely that some SE SNe, with progenitors very similar to those of hydrogen-free $\mathrm{SNe} \mathrm{Ib}$ formed in binary systems, will end up with a small part of the hydrogen envelope remaining and that these should spectroscopically be very similar to $\mathrm{SNe}$ IIb. It could be that iPTF13bvn is one of 
these atypical $\mathrm{SNe} \mathrm{Ib}$, and in that case it should perhaps be considered to be a SN IIb. It was also pointed out that iPTF13bvn is a clear outlier in terms of the $\mathrm{H} \alpha$ absorption strength in the sample studied by Liu et al. (2016). However, we should note that we do not see any sign of the Balmer lines in the spectra of iPTF13bvn other than possibly $\mathrm{H} \alpha$.

Regarding our hydrodynamical modeling, there is always a concern that the ejecta masses of $\mathrm{SNe} \mathrm{IIb} / \mathrm{Ib}$ could be underestimated from the LC-peak widths, owing to significant fallback onto a central black hole. However, in such a picture it becomes difficult to explain the oxygen masses derived from the late-time [OI] $\lambda \lambda 6300,6364$ emission lines. If there is significant fallback, most of the oxygen should fall back into the black hole, but we find that the late-time oxygen emission of both PTF12os and iPTF13bvn is very consistent with low ZAMS-mass stars. The rest of the explosion parameters we find are also very consistent with those of other SNe IIb and SNe Ib in the literature.

Our study of the metallicity in the vicinity of PTF12os and iPTF13bvn shows that both of these $\mathrm{SNe}$ exploded in regions of comparable metallicity, with oxygen abundance very close to the solar value. A solar metallicity is consistent with the models by Yoon et al. (2010) that predict a small amount of hydrogen remaining around some $\mathrm{SNe} \mathrm{Ib}$.

Eldridge \& Maund (2016) have also constructed a binary model for the iPTF13bvn system based on pre-explosion and late-time (+740 d) HST data, where the progenitor of the SN is constrained to a mass range of $10-12 M_{\odot}$. This is very similar to the mass-constraint from our nebular modeling, and thus it seems that a very consistent picture of this $\mathrm{SN}$ is emerging. These HST observations were likely still dominated by light from the SN. However, it is possible that in the near future the first direct detection of the light from a SN Ib binary companion will be possible via another set of HST observations.

Finally, we stress again that the overall appearance of PTF12os and iPTF13bvn is very similar indeed. Investigating two such SE SNe in the same galaxy has allowed a detailed comparison of subtle differences, but generically both the LCs and the spectral evolution are astonishingly similar (and also to the well-studied SN 2011dh). We note that observations of polarization for both SN 2011dh (Mauerhan et al. 2015b) and iPTF13bvn (Reilly et al. 2016) have suggested significant asymmetry and line-of-sight effects. In terms of the overall observables for these $\mathrm{SNe}$, such effects are not dominant.

Acknowledgements. We gratefully acknowledge support from the Knut and Alice Wallenberg Foundation. The Oskar Klein Centre is funded by the Swedish Research Council. This research used resources of the National Energy Research Scientific Computing Center, a DOE Office of Science User Facility supported by the Office of Science of the US Department of Energy under Contract No. DE-AC02-05CH11231. We acknowledge help from Rob Fesen, Craig Wheeler, and Shazrene Mohamed with the SALT data, as well as Jeffrey Silverman for help with the Keck data. We thank Alastair Bruce for assistance with the WHT observing and data reduction. We especially thank Shri Kulkarni, Mansi Kasliwal, Yi Cao, Anna-Lisa de Cia, Jerod Parrent, Assaf Horesh, Tom Matheson, Melissa Graham, Dan Perley, Eric Bellm, Ofer Yaron, Yen-Chen Pan and Kelsey Clubb for help with observations and/or reductions within the PTF effort. We also acknowledge observers, organizers, and data reducers who participated in the BLP 2012P campaign, in particular Andrea Pastorello, Max Stritzinger, Cosimo Inserra, Flora Cellier-Holzem, Luca Borsato, Valerio Nascimbeni, Stefano Benetti, and Stefan Taubenberger. We thank Doug Leonard for discussions regarding complementary MLO data. This work is partly based on observations obtained with the Nordic Optical Telescope, operated by the Nordic Optical Telescope Scientific Association at the Observatorio del Roque de los Muchachos, La Palma, Spain. We acknowledge the exceptional support we received from the NOT staff throughout this campaign. Also based in part on observations made with the Gran Telescopio Canarias (GTC), installed in the Spanish Observatorio del Roque de los Muchachos of the Instituto de Astrofisica de Canarias, in the island of La Palma. This work is based in part on observations from the LCOGT network. Some of the data presented herein were obtained at the W. M. Keck Observatory, which is operated as a scientific partnership among the California Institute of Technology, the University of California, and NASA; the observatory was made possible by the generous financial support of the W. M. Keck Foundation. Research at Lick Observatory is partially supported by a generous gift from Google. CAFOS, AFOSC, and EFOSC2 data were taken within the European supernova collaboration involved in the ESO-NTT large programme 184.D-1140 led by Stefano Benetti. Partially based on observations collected at Copernico telescope (Asiago, Italy) of the INAF - Osservatorio Astronomico di Padova, and the $2.2 \mathrm{~m}$ Telescope of the Centro Astronomico Hispano-Aleman, Calar Alto, Spain. Based in part on observations obtained at the Gemini Observatory, which is operated by the Association of Universities for Research in Astronomy, Inc., under a cooperative agreement with the NSF on behalf of the Gemini partnership: the National Science Foundation (United States), the National Research Council (Canada), CONICYT (Chile), Ministerio de Ciencia, Tecnología e Innovación Productiva (Argentina), and Ministério da Ciência, Tecnologia e Inovação (Brazil). The Hobby-Eberly Telescope (HET) is a joint project of the University of Texas at Austin, the Pennsylvania State University, Stanford University, Ludwig-Maximilians-Universität München, and Georg-August-Universität Göttingen. The HET is named in honor of its principal benefactors, William P. Hobby and Robert E. Eberly. Some of the observations reported in this paper were obtained with the Southern African Large Telescope (SALT). This paper is partly based on observations made with the Italian Telescopio Nazionale Galileo (TNG) operated on the island of La Palma by the Fundación Galileo Galilei of the INAF (Istituto Nazionale di Astrofisica) at the Spanish Observatorio del Roque de los Muchachos of the Instituto de Astrofisica de Canarias. Partially based on observations obtained with the Apache Point Observatory 3.5-m telescope, which is owned and operated by the Astrophysical Research Consortium. This paper includes data gathered with the $6.5 \mathrm{~m}$ Magellan Telescopes located at Las Campanas Observatory, Chile. Based in part on observations made with ESO Telescopes at the La Silla Paranal Observatory under programme 093.D-0199(A). We are grateful for the assistance of the staff members at all observatories where we obtained data. A.G.-Y. is supported by the EU/FP7 via ERC grant No. 307260, the Quantum Universe I-Core program by the Israeli Committee for Planning and Budgeting; by Minerva and ISF grants; by the Weizmann-UK making connections program; and by Kimmel and ARCHES awards. A.V.F.'s research is supported by NASA/HST grant AR-14295 from the Space Telescope Science Institute, which is operated by the Association of Universities for Research in Astronomy, Inc., under NASA contract NAS5-26555; additional financial assistance was provided by the Christopher R. Redlich Fund, the TABASGO Foundation, and NSF grant AST-1211916. N.E.R. is supported by the PRIN-INAF 2014 with the project: Transient Universe: unveiling new types of stellar explosions with PESSTO. This work was partly supported by the European Union FP7 program though ERC grant number 320360. We acknowledge funding from the European Research Council under the European Union's Seventh Framework Programme (FP7/2007-2013)/ERC Grant agreement $n^{\circ}$ [291222].

\section{References}

Ahn, C. P., Alexandroff, R., Allende Prieto, C., et al. 2014, ApJS, 211, 17 Arcavi, I., Gal-Yam, A., Yaron, O., et al. 2011, ApJ, 742, L18 Arcavi, I., Gal-Yam, A., Ben-Ami, S., et al. 2012, ATel, 3881, 1 Arcavi, I., Ofek, E., Cao, Y., et al. 2013a, ATel, 5152, 1

Arcavi, I., Yaron, O., Gal-Yam, A., \& Cao, Y. 2013b, ATel, 5140, 1 Asplund, M., Grevesse, N., Sauval, A. J., \& Scott, P. 2009, ARA\&A, 47, 481 Benvenuto, O. G., Bersten, M. C., \& Nomoto, K. 2013, ApJ, 762, 74 Bersten, M. C., Benvenuto, O. G., Nomoto, K., et al. 2012, ApJ, 757, 31 Bersten, M. C., Benvenuto, O. G., Folatelli, G., et al. 2014, AJ, 148, 68 Bertin, E. 2013, Astrophysics Source Code Library [record ascl:1301.001] Bertin, E., \& Arnouts, S. 1996, A\&AS, 117, 393

Blondin, S., Mandel, K. S., \& Kirshner, R. P. 2011, A\&A, 526, A81 Brown, P. J., Holland, S. T., Immler, S., et al. 2009, AJ, 137, 4517 Brown, T. M., Baliber, N., Bianco, F. B., et al. 2013, PASP, 125, 1031 Cano, Z. 2013, MNRAS, 434, 1098

Cao, Y., Kasliwal, M. M., Arcavi, I., et al. 2013, ApJ, 775, L7

Cardelli, J. A., Clayton, G. C., \& Mathis, J. S. 1989, ApJ, 345, 245

Cenko, S. B., Fox, D. B., Moon, D.-S., et al. 2006, PASP, 118, 1396

Claeys, J. S. W., de Mink, S. E., Pols, O. R., Eldridge, J. J., \& Baes, M. 2011, A\&A, 528, A131

Conti, P. 1976, Mem. Soc. Roy. Sci. Liège, 9, 193

Dessart, L., \& Hillier, D. J. 2005, A\&A, 439, 671

Dessart, L., Hillier, D. J., Livne, E., et al. 2011, MNRAS, 414, 2985

Dessart, L., Hillier, D. J., Li, C., \& Woosley, S. 2012, MNRAS, 424, 2139

de Vaucouleurs, G., de Vaucouleurs, A., Corwin, Jr., H. G., et al. 1991, Third Reference Catalogue of Bright Galaxies, Volume I: Explanations and references. Volume II: Data for galaxies between $0^{\mathrm{h}}$ and $12^{\mathrm{h}}$, Volume III: Data for galaxies between $12^{\mathrm{h}}$ and $24^{\mathrm{h}}$ (Springer) 
Dimai, A., Briganti, F., \& Brimacombe, J. 2012, Central Bureau Electronic Telegrams, 2993, 1

Dolphin, A. E. 2000, PASP, 112, 1383

Eldridge, J. J., \& Maund, J. R. 2016, MNRAS, 461, L117

Eldridge, J. J., \& Stanway, E. R. 2009, MNRAS, 400, 1019

Eldridge, J. J., Fraser, M., Smartt, S. J., Maund, J. R., \& Crockett, R. M. 2013, MNRAS, 436, 774

Eldridge, J. J., Fraser, M., Maund, J. R., \& Smartt, S. J. 2015, MNRAS, 446 2689

Ergon, M., Sollerman, J., Fraser, M., et al. 2014, A\&A, 562, A17

Ergon, M., Jerkstrand, A., Sollerman, J., et al. 2015, A\&A, 580, A142

Faber, S. M., Phillips, A. C., Kibrick, R. I., et al. 2003, in Instrument Design and Performance for Optical/Infrared Ground-based Telescopes, eds. M. Iye, \& A. F. M. Moorwood, SPIE Conf. Ser., 4841, 1657

Falk, S. W., \& Arnett, W. D. 1977, ApJS, 33, 515

Filippenko, A. V. 1997, ARA\&A, 35, 309

Folatelli, G., Bersten, M. C., Benvenuto, O. G., et al. 2014a, ApJ, 793, L22

Folatelli, G., Bersten, M. C., Kuncarayakti, H., et al. 2014b, ApJ, 792, 7

Folatelli, G., Van Dyk, S. D., Kuncarayakti, H., et al. 2016, ApJ, 825, L22

Fox, O. D., Azalee Bostroem, K., Van Dyk, S. D., et al. 2014, ApJ, 790, 17

Fremling, C., Sollerman, J., Taddia, F., et al. 2014, A\&A, 565, A114

Gal-Yam, A., Maoz, D., Guhathakurta, P., \& Filippenko, A. V. 2008, ApJ, 680, 550

Gehrels, N., Chincarini, G., Giommi, P., et al. 2004, ApJ, 611, 1005

Groh, J. H., Georgy, C., \& Ekström, S. 2013, A\&A, 558, L1

Gusev, A. S., Pilyugin, L. S., Sakhibov, F., et al. 2012, MNRAS, 424, 1930

Hachinger, S., Mazzali, P. A., Taubenberger, S., et al. 2012, MNRAS, 422, 70

Iben, Jr., I., \& Tutukov, A. V. 1985, ApJS, 58, 661

Jerkstrand, A., Ergon, M., Smartt, S. J., et al. 2015, A\&A, 573, A12

Jerkstrand, A., Ergon, M., Smartt, S., et al. 2016, A\&A, submitted

Jester, S., Schneider, D. P., Richards, G. T., et al. 2005, AJ, 130, 873

Kankare, E., Kotak, R., Pastorello, A., et al. 2015, A\&A, 581, L4

Kelly, P. L., \& Kirshner, R. P. 2012, ApJ, 759, 107

Kuncarayakti, H., Maeda, K., Bersten, M. C., et al. 2015, A\&A, 579, A95

Landolt, A. U. 1992, AJ, 104, 340

Law, N. M., Kulkarni, S. R., Dekany, R. G., et al. 2009, PASP, 121, 1395

Leitherer, C., Schaerer, D., Goldader, J. D., et al. 1999, ApJS, 123, 3

Li, W., Leaman, J., Chornock, R., et al. 2011, MNRAS, 412, 1441

Liu, Y.-Q., Modjaz, M., Bianco, F. B., \& Graur, O. 2016, ApJ, 827, 90

Lyman, J., Bersier, D., James, P., et al. 2016, MNRAS, 457, 328

Maíz-Apellániz, J. 2004, PASP, 116, 859

Mauerhan, J. C., Van Dyk, S. D., Graham, M. L., et al. 2015a, MNRAS, 447, 1922

Mauerhan, J. C., Williams, G. G., Leonard, D. C., et al. 2015b, MNRAS, 453, 4467

Maund, J. R., \& Smartt, S. J. 2009, Science, 324, 486

Maund, J. R., Arcavi, I., Ergon, M., et al. 2015, MNRAS, 454, 2580
Mazzali, P. A., Foley, R. J., Deng, J., et al. 2007, ApJ, 661, 892

Mazzali, P. A., Maurer, I., Valenti, S., Kotak, R., \& Hunter, D. 2010, MNRAS, 408, 87

Milisavljevic, D., Fesen, R., Pickering, T., et al. 2013a, ATel, 5142, 1

Milisavljevic, D., Margutti, R., Soderberg, A. M., et al. 2013b, ApJ, 767, 71

Ofek, E. O. 2014, Astrophysics Source Code Library [record ascl: 1407.005]

Parrent, J. T., Milisavljevic, D., Soderberg, A. M., \& Parthasarathy, M. 2016, ApJ, 820, 75

Pastorello, A., Kasliwal, M. M., Crockett, R. M., et al. 2008, MNRAS, 389, 955

Paxton, B., Bildsten, L., Dotter, A., et al. 2010, MESA: Modules for Experiments in Stellar Astrophysics (Astrophysics Source Code Library)

Pettini, M., \& Pagel, B. E. J. 2004, MNRAS, 348, L59

Pilyugin, L. S., Vílchez, J. M., \& Contini, T. 2004, A\&A, 425, 849

Piro, A. L., \& Nakar, E. 2013, ApJ, 769, 67

Poznanski, D., Ganeshalingam, M., Silverman, J. M., \& Filippenko, A. V. 2011, MNRAS, 415, L81

Poznanski, D., Prochaska, J. X., \& Bloom, J. S. 2012, MNRAS, 426, 1465

Reilly, E., Maund, J. R., Baade, D., et al. 2016, MNRAS, 457, 288

Roming, P. W. A., Hunsberger, S. D., Mason, K. O., et al. 2004, in X-Ray and Gamma-Ray Instrumentation for Astronomy XIII, eds. K. A. Flanagan, \& O. H. W. Siegmund, SPIE Conf. Ser., 5165, 262

Roming, P. W. A., Kennedy, T. E., Mason, K. O., et al. 2005, Space Sci. Rev., 120,95

Schlafly, E. F., \& Finkbeiner, D. P. 2011, ApJ, 737, 103

Silverman, J. M., Kong, J. J., \& Filippenko, A. V. 2012, MNRAS, 425, 1819

Simcoe, R. A., Burgasser, A. J., Schechter, P. L., et al. 2013, PASP, 125, 270

Smartt, S. J., Eldridge, J. J., Crockett, R. M., \& Maund, J. R. 2009, MNRAS, 395, 1409

Smith, N. 2014, ARA\&A, 52, 487

Srivastav, S., Anupama, G. C., \& Sahu, D. K. 2014, MNRAS, 445, 1932

Strotjohann, N. L., Ofek, E. O., Gal-Yam, A., et al. 2015, ApJ, 811, 117

Taddia, F., Sollerman, J., Razza, A., et al. 2013, A\&A, 558, A143

Taddia, F., Sollerman, J., Leloudas, G., et al. 2015, A\&A, 574, A60

Taubenberger, S., Navasardyan, H., Maurer, J. I., et al. 2011, MNRAS, 413, 2140

Tully, R. B., Rizzi, L., Shaya, E. J., et al. 2009, AJ, 138, 323

Tully, R. B., Courtois, H. M., Dolphin, A. E., et al. 2013, AJ, 146, 86

Turatto, M., Benetti, S., \& Cappellaro, E. 2003, in From Twilight to Highlight: The Physics of Supernovae, eds. W. Hillebrandt, \& B. Leibundgut, 200

Van Dyk, S. D., Li, W., Cenko, S. B., et al. 2011, ApJ, 741, L28

Van Dyk, S. D., Gal-Yam, A., Arcavi, I., et al. 2012, ATel, 3884, 1

Vinkó, J., Sárneczky, K., Balog, Z., et al. 2009, ApJ, 695, 619

Wheeler, J. C., Johnson, V., \& Clocchiatti, A. 2015, MNRAS, 450, 1295

Woosley, S. E., \& Heger, A. 2007, Phys. Rep., 442, 269

Yaron, O., \& Gal-Yam, A. 2012, PASP, 124, 668

Yoon, S.-C. 2015, PASA, 32, 15

Yoon, S.-C., Woosley, S. E., \& Langer, N. 2010, ApJ, 725, 940 


\section{Appendix A: Additional figures and tables}

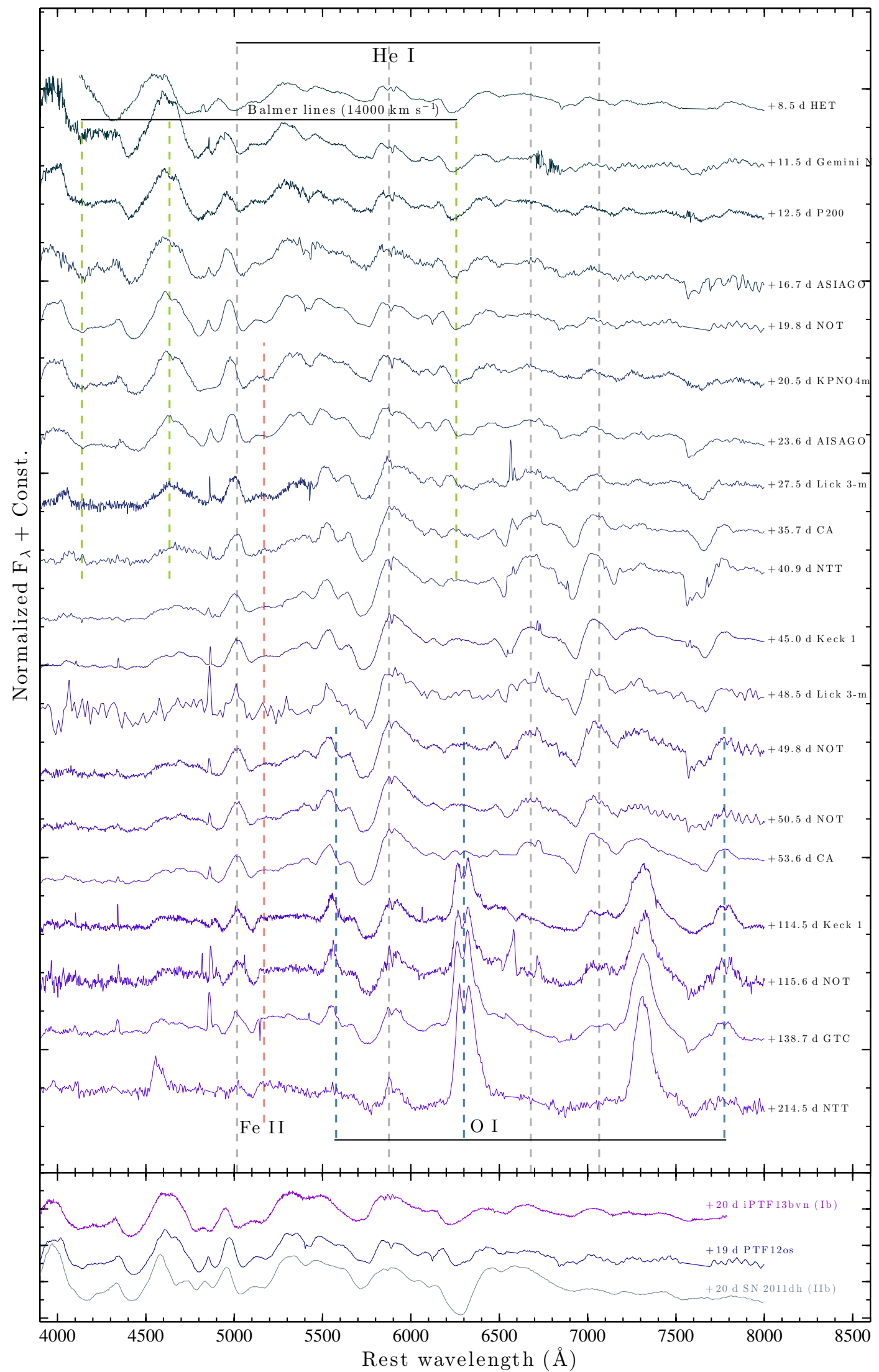

Fig. A.1. Spectral sequence of PTF12os (SN 2012P) (top panel). Comparison of the visible spectra of PTF12os to iPTF13bvn (Ib) and SN 2011dh (IIb) at approximately $+20 \mathrm{~d}$ (bottom panel). Thick dashed lines mark the central wavelength of the marked emission lines at rest, except for the Balmer lines, which are marked by green dashed lines at $14000 \mathrm{~km} \mathrm{~s}^{-1}$. Telluric features near the wavelengths $6860 \AA$ and especially $7600 \AA$ are present in some of the spectra. 


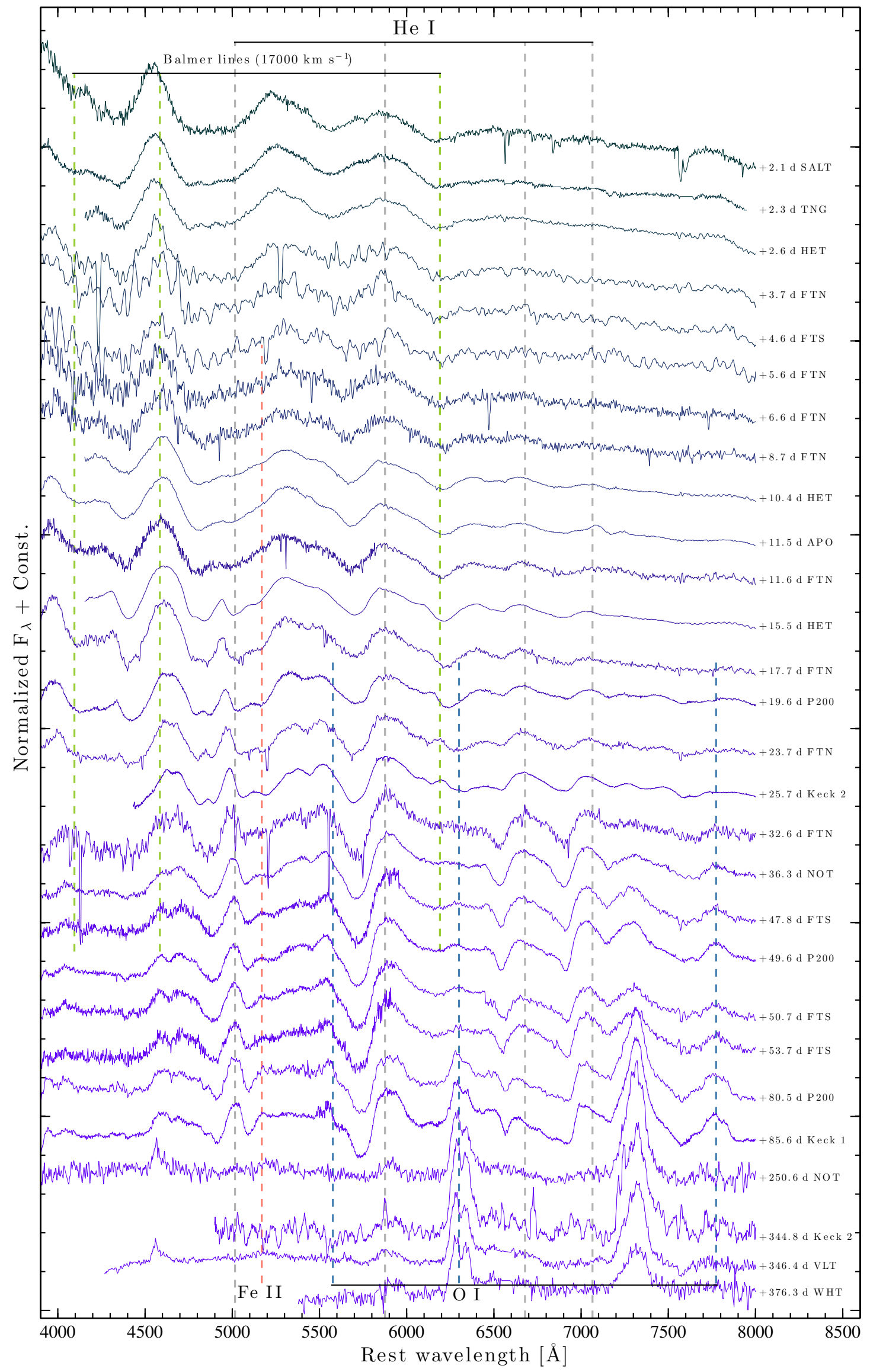

Fig. A.2. Spectral sequence of iPTF13bvn. Thick dashed lines mark the central wavelength of the marked emission lines at rest, except for the Balmer lines, which are marked by green dashed lines at $17000 \mathrm{~km} \mathrm{~s}^{-1}$. Telluric features near the wavelengths $6860 \AA$ and especially $7600 \AA$ are present in some of the spectra. 
A\&A 593, A68 (2016)
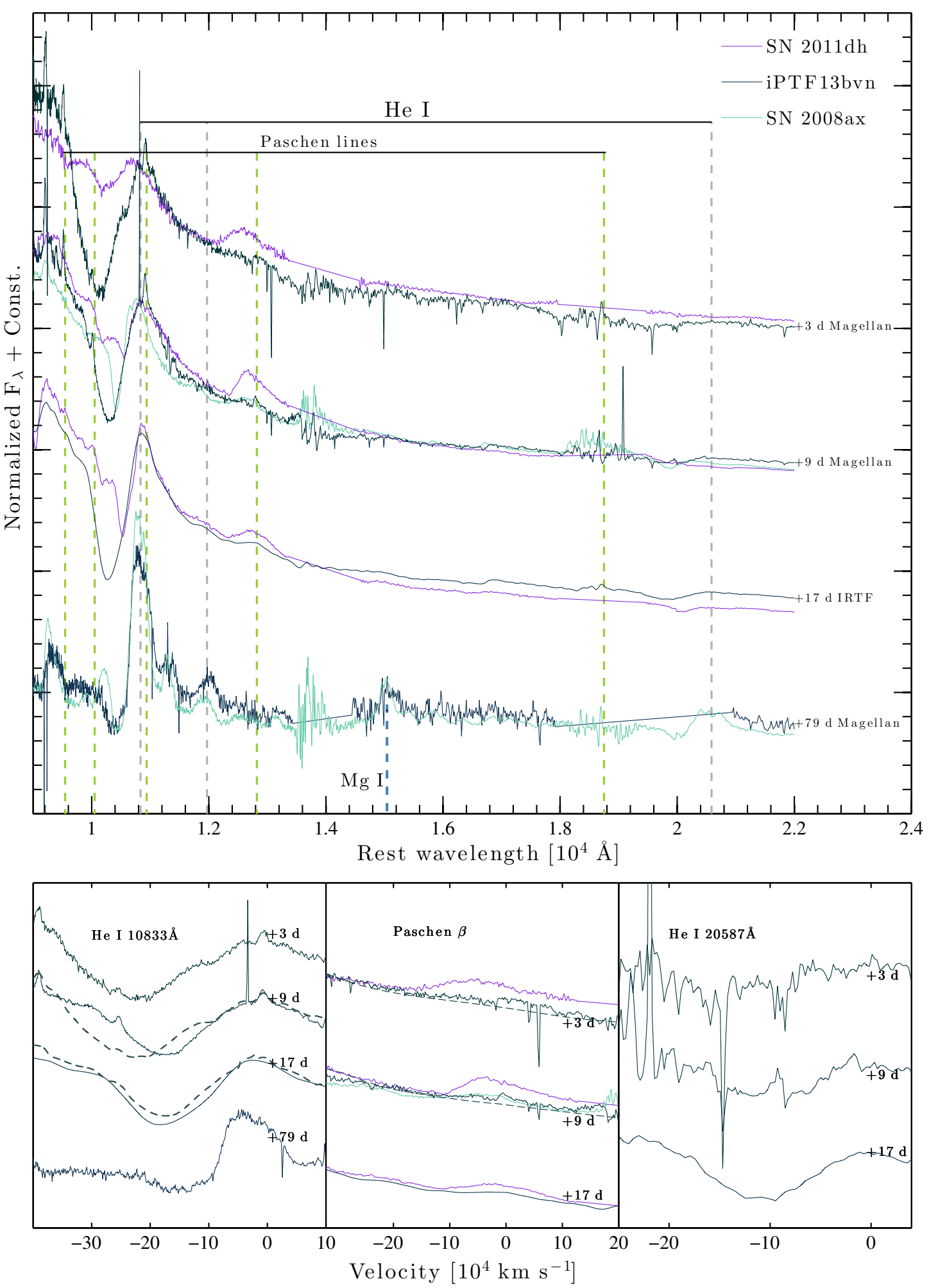

Fig. A.3. Near-infrared spectral sequence of iPTF13bvn (black lines) compared to SN 2011dh (purple lines) and SN 2008ax (cyan lines) at similar epochs. The spectra have been normalized using the median and shifted by a constant between the epochs. The top panel shows the spectral sequence in wavelength space. In the bottom panels we highlight small sections in velocity space. The bottom-left panel displays the region around He I $\lambda 10$ 830; the dashed lines show a smoothed version of the previous spectra in the sequence to highlight the evolution of the line. The bottomcenter panel emphasizes the region around Paschen $\beta$; the dashed lines show heavily smoothed versions of the spectra of iPTF13bvn, which should give a rough estimate of the continuum. The bottom-right panel reveals the region around He I 120590. 
Table A.1. Log of the spectral observations of PTF12os.

\begin{tabular}{lccc}
\hline \hline $\begin{array}{l}\text { Date } \\
\text { [2012] }\end{array}$ & JD $-2,455,933.00$ & Telescope+Instrument & $\begin{array}{c}\text { Range } \\
{[\AA]}\end{array}$ \\
\hline 14 Jan. & +8.5 & HET+LRS & $4148-10403$ \\
17 Jan. & +11.5 & GEMINI N.+GMOS & $3500-9654$ \\
18 Jan. & +12.5 & P200+DBSP & $3470-10200$ \\
22 Jan. & +16.7 & ASIAGO 1.82m+AFOSC & $3882-8180$ \\
25 Jan. & +19.8 & NOT+ALFOSC & $3560-9086$ \\
26 Jan. & +20.5 & KPNO 4m+RC Spec & $3500-8450$ \\
29 Jan. & +23.6 & ASIAGO 1.82m+AFOSC & $3892-8180$ \\
02 Feb. & +27.5 & Lick 3m+Kast & $3500-10000$ \\
10 Feb. & +35.7 & CA-2.2+CAFOS & $3877-10120$ \\
15 Feb. & +40.9 & NTT+EFOSC & $3910-9295$ \\
20 Feb. & +45.0 & Keck 1+LRIS & $3830-10250$ \\
23 Feb. & +48.5 & Lick 3m+Kast & $3504-10152$ \\
24 Feb. & +49.8 & NOT+ALFOSC & $3974-9153$ \\
25 Feb. & +50.5 & NOT+ALFOSC & $3402-9129$ \\
28 Feb. & +53.6 & CA-2.2+CAFOS & $3877-10120$ \\
29 Apr. & +114.5 & Keck 1+LRIS & $3299-10250$ \\
30 Apr. & +115.6 & NOT+ALFOSC & $3974-8999$ \\
23 May & +138.7 & GTC+OSIRIS & $3600-10000$ \\
08 Aug. & +214.5 & NTT+EFOSC & $3910-9295$ \\
\hline
\end{tabular}

Table A.2. Log of the spectral observations of iPTF13bvn.

\begin{tabular}{|c|c|c|c|c|}
\hline Date & $\begin{array}{c}\text { Phase } \\
\text { JD }-2456459.17\end{array}$ & Telescope+Instrument & $\begin{array}{c}\text { Range } \\
{[\AA]}\end{array}$ & Reference \\
\hline 17 June 2013 & +2.15 & SALT+Spectrograph & $3500-9000$ & This work \\
\hline 17 June 2013 & +2.3 & TNG+DOLORES & $3164-7987$ & 1 \\
\hline 18 June 2013 & +2.6 & HET+LRS & $4000-11000$ & 1 \\
\hline 18 June 2013 & +3.4 & Magellan Baade+FIRE & $9000-25000$ & 1 \\
\hline 19 June 2013 & +3.7 & FTN+FLOYDS & $3179-11038$ & 1 \\
\hline 20 June 2013 & +4.6 & FTS+FLOYDS & $3179-11038$ & 1 \\
\hline 21 June 2013 & +5.6 & FTN+FLOYDS & $3179-11038$ & 1 \\
\hline 22 June 2013 & +6.7 & FTN+FLOYDS & $3179-11038$ & 1 \\
\hline 24 June 2013 & +8.7 & FTN+FLOYDS & $3179-11038$ & 1 \\
\hline 24 June 2013 & +8.5 & Magellan Baade+FIRE & $9000-25000$ & 1 \\
\hline 26 June 2013 & +10.4 & HET+LRS & $4172-10800$ & 1 \\
\hline 27 June 2013 & +11.5 & APO+DIS & $3338-10039$ & 1 \\
\hline 27 June 2013 & +11.7 & FTN+FLOYDS & $3179-11038$ & 1 \\
\hline 01 July 2013 & +15.5 & HET+LRS & $4172-10800$ & 1 \\
\hline 02 July 2013 & +16.7 & IRTF+SpeX & $9000-25000$ & 1 \\
\hline 03 July 2013 & +17.7 & FTN+FLOYDS & $3179-11038$ & This work \\
\hline 05 July 2013 & +19.6 & P200+DBSP & $3200-10100$ & 2 \\
\hline 09 July 2013 & +23.7 & FTN+FLOYDS & $3179-11038$ & This work \\
\hline 11 July 2013 & +25.7 & Keck 2+DEIMOS & $4450-9625$ & 2 \\
\hline 18 July 2013 & +32.6 & FTN+FLOYDS & $3179-11038$ & This work \\
\hline 21 July 2013 & +36.3 & NOT+ALFOSC & $3601-9141$ & 2 \\
\hline 02 Aug. 2013 & +47.8 & FTS+FLOYDS & $3179-11038$ & This work \\
\hline 04 Aug. 2013 & +49.6 & P200+DBSP & $3200-10100$ & 2 \\
\hline 05 Aug. 2013 & +50.7 & FTS+FLOYDS & $3179-11038$ & This work \\
\hline 08 Aug. 2013 & +53.7 & FTS+FLOYDS & $3179-11038$ & This work \\
\hline 02 Sep. 2013 & +78.7 & Magellan Baade+FIRE & $9000-25000$ & This work \\
\hline 04 Sep. 2013 & +80.5 & P200+DBSP & $3200-10100$ & 2 \\
\hline 09 Sep. 2013 & +85.6 & Keck 1+LRIS & $3200-10000$ & 2 \\
\hline 21 Feb. 2014 & +250.6 & NOT+ALFOSC & $3301-9142$ & This work \\
\hline 26 May 2014 & +344.8 & Keck 2+DEIMOS & $4915-10134$ & This work \\
\hline 28 May 2014 & +346.4 & VLT+FORS2 & $3300-11000$ & This work \\
\hline 26 June 2014 & +376.3 & WHT+ISIS & $5400-9000$ & This work \\
\hline
\end{tabular}

References. 1. Cao et al. (2013); 2. Fremling et al. (2014). 